\title{
Historical and temporal trends of climatic parameters in North East India
}

\author{
Lairenjam C. ${ }^{1}$, Hodam S. ${ }^{2}$, Bandyopadhyay A. ${ }^{3,}{ }^{*}$ and Bhadra A. ${ }^{3}$ \\ ${ }^{1}$ Department of Agricultural Engineering and Technology, School of Engineering and Technology (SET), Nagaland University, Dimapur \\ 797112, Nagaland, India \\ ${ }^{2}$ RMO Division, National Institute of Hydrology, Roorkee 247667, Uttarakhand, India \\ ${ }^{3}$ Department of Agricultural Engineering, North Eastern Regional Institute of Science and Technology, Nirjuli (Itanagar) 791109, \\ Arunachal Pradesh, India \\ Received: 12/06/2017, Accepted: 18/09/2017, Available online: 25/10/2017 \\ *to whom all correspondence should be addressed: e-mail: arnabbandyo@yahoo.co.in
}

\section{Abstract}

Accessing temporal trend of different meteorological parameters is essential for understanding the local climate changing pattern of a region. Quantitative estimates of the effect of climate change helps in understanding, planning, and management of water resources systems. In this study, monthly meteorological data were collected from 30 stations of north-east (NE) India for 1971-2010 and nonparametric Mann-Kendall (MK) test and Sen slope were employed for detection and quantification of significant temporal trends, respectively. An ESRI ArcGIS toolbar "ArcTrends" was used for the above mentioned tasks. The results obtained for rainfall were of mixed nature and both increasing and decreasing significant trends were found for different stations in different months. Most of the negative trends were found in the months of July-August (monsoon), whereas, more stations showed positive trends in April-May (pre-monsoon), and October-November (post-monsoon), indicating inter-seasonal shifting of rainfall without much change in the annual total. Number of rainy days was found to have positive trends in MarchMay (pre-monsoon) and negative trends in SeptemberDecember. Except some positive trends during JuneDecember in Manipur and Meghalaya, there were no significant trends in maximum temperature. In some stations, minimum temperature was found to have significant increasing trends throughout the year indicating a general rising trend in NE India. Some major towns like Guwahati, Imphal, Agartala and Kailashshahar showed significant positive trends in mean temperature, mostly during June-December. Mean relative humidity was, in general, found to be significantly increasing, especially during February-March. In some stations, wind speed was found to have significant negative trends throughout the year, with Agartala being the most affected.

Keywords: Temporal trend, Mann-Kendall (MK) test, Sen slope, north-east India, Brahmaputra and Barak basin

\section{Introduction}

Accessing the temporal trend in different meteorological parameters is essential for understanding the local climate changing pattern of a region. It helps in enhancing the adaptive capacity of the society by forecasting the hydrologic and agricultural effects of changing climate. The third assessment report of the Intergovernmental Panel on Climate Change (IPCC, 2001) outlines the general impacts of climate change on water resources. It indicates an intensification of the global hydrologic cycle affecting both ground and surface water availability. IPCC, in its fourth assessment report (IPCC, 2007), observed that natural systems in all continents and most oceans were affected by regional climate change, particularly increase in temperature. Understanding the dynamic nature of climate is vital for planning and management of the available water resources. Regional climatic events often cause catastrophic damage to unprepared human societies and, therefore, regional assessments are needed for minimizing the damage. Hence, quantitative estimates of effect of climate change on individual meteorological variables are essential for planning and management of the future water resources systems.

In the past decades, different meteorological parameters like rainfall, temperature, etc., have been studied and analyzed by researchers and scientists around the globe. In India, Paramanik and Jagannathan (1954) found that the rise in annual mean surface temperature over India is in agreement with the reported rise of global surface temperature. Hingane et al., (1985), Srivastava et al., (1992), Rupakumar et al., (1994), De and Mukhopadhyay (1998), Pant et al., (1999), Singh et al., (2001), and Singh and Sontakke, (2002) also reported increasing trend in surface temperature in different parts of India. Bandyopadhyay et al., (2009) reported significant decreasing trend in grass reference evapotranspiration over entire India caused by increasing relative humidity and decreasing wind speed, without any general increasing trend in rainfall. Udo-Inyang and Edem, (2012) stated that rainfall trend was an important characteristics of rainfall that varied both in time and space. Nema et al., (2014) 
observed a generalized negative trend in rainfall for the entire Bist-Doab region of Punjab.

In north-east (NE) India, no significant trend in annual regional rainfall was reported except for south Assam meteorological subdivision, where monsoonal rainfall was found to be decreasing (Mirza et al., 1998; Goswami and Das, 2003; Das, 2004). Pant and Rupakumar, (1997) and Das (2004), however, reported an increasing trend in the annual average temperatures (maximum and mean).

The statistical tools to analyze temporal trends range from simple linear regression to non-parametric tests. Nonparametric tests are robust, unaffected by presence of missing values or outliers, and need no probability distribution of the variate to be assumed. Among the nonparametric tests, Mann-Kendall (MK) test and Sen's slope are widely used for detecting statistically significant trend and finding magnitude of trend, respectively, in a time series. Since the first proposals of the MK test by Mann (1945) and Kendall (1975), covariance between MK statistics was proposed by Dietz and Kileen (1981) and the test was extended to include seasonality (Hirsch et al., 1982), multiple monitoring sites and variates (Lettenmaier, 1988) and presence of covariates representing natural fluctuations (Libiseller and Grimvall, 2002). Hamed and Rao (1998) presented theoretical results about the evaluation of the mean and variance of the MK test statistic in the presence of auto-correlation.

Therefore, in this study, an attempt has been made to detect and quantify the temporal trend of climatic variables for meteorological stations situated in north eastern region of India. The Mann-Kendall (MK) test (Mann 1945, Kendall 1975) with modified effective sample size (ESS) approach for serially correlated data (Yue and Wang 2004), and Sen slope (Sen 1968) have been used for this purpose.

\section{Data and methodology}

\subsection{Study Area}

The NE India comprises of the states of Arunachal Pradesh, Assam, Manipur, Meghalaya, Mizoram, Nagaland, Sikkim, and Tripura (Fig. 1). The region stretches between $21^{\circ} 50^{\prime}$ and $29^{\circ} 34^{\prime} \mathrm{N}$ latitudes, and $85^{\circ} 34^{\prime}$ and $97^{\circ} 50^{\prime} \mathrm{E}$ longitudes. The region has a geographical area of 2,55,168 $\mathrm{km}^{2}$ (25.52 M ha), which is $8 \%$ of the area of India. In this study, Sikkim was excluded due to spatial discontinuity.

The NE India is known for its rich biodiversity, heavy precipitation, and high seismicity. High variation in the physiological features and huge elevation differences are responsible for its varied climate. The rainfall in the region is mainly contributed by south-west monsoon and is highly seasonal. Agricultural products play an important role for the economy of NE India and any change in the spatial and temporal pattern of rainfall is expected to directly affect the monsoon-reliant agricultural and forest ecosystems (Das et al., 2009). The region experiences great variation in the temperature ranges also. Hence, the NE India, exhibits all forms of physiographic features ranging from high Himalayan mountains to low foothills, from elevated platues to extremely flat lowland basins and flood plains. Physiography is the prime factor in determining the mesoscale distribution and variability of rainfall in the region.

Global warming is affecting NE India in many ways. It is vulnerable to water-related disasters because of its proximity to fragile geo environment of eastern Himalaya and economic underdevelopment. The Brahmaputra and the Barak (Meghna) river systems act as sources of both resource and vulnerability. As agriculture mainly thrives on monsoon rainwater, it is likely to be affected badly due to the changing rainfall pattern. The cropping pattern may have to be redesigned even. Therefore, under global warming conditions, the temporal variation of climatic variables in NE India needs to be assessed to address the resulting impact on water resources.

\subsection{Meteorological Data}

Monthly meteorological data for 40 years (1971-2010) were collected from India Meteorological Department (IMD), State Govt. departments and other Central Govt. organizations. The meteorological data were collected from 30 stations of NE India which included maximum, minimum and mean temperatures; mean relative humidity; wind speed; number of rainy days; and rainfall. Table 1 and Fig. 1 show the list and locations of the meteorological stations along with the availability of meteorological data.

\subsection{Determination of Temporal Trends of Meteorological Parameters}

The trend analyses were performed on monthly and yearly average (annual) data to determine the existence and magnitude of any statistically significant trend in them over the time period considered. It was done for individual stations, state-wise, and for entire NE region as a whole. For state-wise analysis, the average of each parameter from all the stations in the particular state was considered, whereas, the average of each parameter from all the stations under study was considered in case of the whole region. The MK test was employed for detection of trend whereas Sen slope was used to quantify the magnitude of such trend.

\subsubsection{MK test}

The MK test (Mann, 1945; Kendall, 1975) statistic $S$ is defined as:

$$
S=\sum_{i=1}^{n-1} \sum_{j=i+1}^{n} \operatorname{sgn}\left(x_{j}-x_{i}\right)
$$

where, $n$ is the length of the dataset, $x_{i}$ and $x_{j}$ are the $i^{\text {th }}$ and $j^{\text {th }}$ data in the series $(\forall i<j)$ and

$$
\operatorname{sgn}\left(x_{j}-x_{i}\right)=\left\{\begin{array}{cc}
1, & x_{j}>x_{i} \\
0, & x_{j}=x_{i} \\
-1, & x_{j}<x_{i}
\end{array}\right.
$$

For $n \geq 10, S$ is normally distributed with mean $=0$ and variance,

$$
\sigma_{s}^{2}=\frac{n(n-1)(2 n+5)-\sum_{k=1}^{m} t_{k}\left(t_{k}-1\right)\left(2 t_{k}+5\right)}{18}
$$


Table 1. Station details and data availability status for different meteorological variables (1971-2010)

\begin{tabular}{|c|c|c|c|c|c|c|c|c|c|c|c|c|}
\hline $\begin{array}{l}\text { SI. } \\
\text { No. }\end{array}$ & Station & State* & $\begin{array}{c}\text { Latitude } \\
\left({ }^{\circ} \mathrm{N}\right)\end{array}$ & $\begin{array}{c}\text { Longitude } \\
\left({ }^{\circ} \mathrm{E}\right)\end{array}$ & $\begin{array}{c}\text { Elevation } \\
(\mathrm{m})\end{array}$ & Rainfall & $\begin{array}{c}\text { No. of rainy } \\
\text { days }\end{array}$ & $\begin{array}{c}\text { Max. } \\
\text { temperature }\end{array}$ & $\begin{array}{c}\text { Min. } \\
\text { temperature }\end{array}$ & $\begin{array}{c}\text { Mean } \\
\text { temperature }\end{array}$ & Mean RH & Wind speed \\
\hline 1 & Aalo & \multirow{8}{*}{ AP } & 28.17 & 94.80 & 266 & $(1993-2007)$ & NA & $(1992-2007)$ & (1992-2007) & $(1992-2007)$ & (1993-2007) & NA \\
\hline 2 & Basar & & 27.97 & 94.68 & 578 & (1993-2007) & NA & $(1993-2007)$ & (1993-2007) & $(1993-2007)$ & (1993-2007) & NA \\
\hline 3 & Daporijo & & 27.98 & 94.22 & 248 & (1992-2007) & NA & NA & NA & NA & NA & NA \\
\hline 4 & Itanagar & & 27.00 & 93.07 & 440 & $(1996-2008)$ & (1996-2008) & $(1996-2008)$ & $(1996-2008)$ & $(1996-2008)$ & (1996-2008) & NA \\
\hline 5 & Passighat & & 28.10 & 95.38 & 153 & $(1971-2010)$ & $(1971-2010)$ & $(1971-2010)$ & $(1971-2010)$ & $(1971-2010)$ & (1971-2010) & NA \\
\hline 6 & Roing & & 28.13 & 95.83 & 396 & $(1992-2008)$ & NA & $(1992-2008)$ & NA & NA & (1998-2000) & NA \\
\hline 7 & Seppa & & 27.32 & 93.00 & 363 & (1992-2008) & (1993-2008) & (1992-2008) & (1992-2008) & $(1992-2008)$ & NA & NA \\
\hline 8 & Ziro & & 27.32 & 93.80 & 1688 & $(1971-1980)$ & $(1971-1980)$ & $(1971-1980)$ & $(1971-1980)$ & $(1971-1980)$ & (1971-1980) & (1971-1976) \\
\hline 9 & Chaparmukh & \multirow{11}{*}{ AS } & 26.20 & 95.52 & 66 & $(1971-1992)$ & (1971-1992) & $(1971-1992)$ & $(1971-1992)$ & $(1971-1992)$ & (1971-1991) & (1971-1991) \\
\hline 10 & Dibrugarh & & 27.48 & 95.02 & 35 & $(1971-2000)$ & $(1971-2000)$ & $(1971-2000)$ & $(1971-2000)$ & $(1971-2000)$ & $(1971-2002)$ & $(1971-2000$ \\
\hline 11 & Dhubri & & 26.02 & 89.98 & 295 & (1971-2010) & (1971-2010) & (1971-2010) & (1971-2010) & (1971-2010) & (1971-2010) & (1971-1992) \\
\hline 12 & Guwahati & & 26.10 & 91.58 & 54 & $(1971-2010)$ & (1971-2010) & $(1971-2010)$ & $(1971-2010)$ & $(1971-2010)$ & (1971-2010) & NA \\
\hline 13 & Jorhat & & 26.73 & 94.17 & 99 & (1996-2010) & NA & (2007-2010) & (2007-2010) & (2007-2010) & (2007-2010) & NA \\
\hline 14 & Kumbhigram & & 24.90 & 92.97 & 81 & (1971-1987) & NA & (1971-1987) & (1971-1987) & (1971-1987) & (1971-1987) & NA \\
\hline 15 & Likabali & & 27.60 & 94.72 & 115 & (1993-2007) & NA & (1992-2007) & (1992-2007) & (1992-2007) & NA & NA \\
\hline 16 & Mohanbari & & 27.48 & 95.02 & 111 & $(2001-2010)$ & $(2001-2010)$ & $(2001-2010)$ & $(2001-2010)$ & $(2001-2010)$ & $(2001-2010)$ & NA \\
\hline 17 & N. Lakhimpur & & 27.23 & 94.12 & 99 & (1971-2010) & (1971-2010) & (1971-2010) & (1971-2010) & (1971-2010) & (1971-2010) & NA \\
\hline 18 & Silchar & & 24.82 & 92.80 & 107 & $(1988-2010)$ & $(1988-2010)$ & $(1988-2010)$ & $(1988-2010)$ & $(1988-2010)$ & $(1988-2010)$ & NA \\
\hline 19 & Tezpur & & 26.65 & 92.78 & 79 & (1971-2010) & (1971-2010) & (1971-2010) & (1971-2010) & (1971-2010) & (1971-2010) & (1971-2001) \\
\hline 20 & Imphal & $\mathrm{MN}$ & 24.77 & 93.90 & 781 & $(1971-2010)$ & $(1971-2010)$ & $(1971-2010)$ & $(1971-2010)$ & $(1971-2010)$ & $(71-10)$ & (1971-1998) \\
\hline 21 & Barapani & \multirow{4}{*}{$\mathrm{ML}$} & 25.70 & 91.97 & 893 & $(1981-2010)$ & (1992-2010) & $(1986-2010)$ & $(1986-2010)$ & $(1986-2010)$ & NA & NA \\
\hline 22 & Cherrapunji & & 25.25 & 91.73 & 1313 & (1971-2010) & (1971-2010) & (1971-2010) & (1971-2010) & (1971-2010) & (1971-2010) & (1971-2001) \\
\hline 23 & Shillong & & 25.57 & 91.88 & 1598 & (1971-2010) & (1971-2010) & (1971-2010) & (1971-2010) & (1971-2010) & (1971-2010) & (1971-2000) \\
\hline 24 & Tura & & 25.52 & 90.23 & 370 & (1971-1985) & (1971-1985) & (1971-1985) & (1971-1985) & (1971-1985) & (1971-1985) & (1971-1985) \\
\hline 25 & Aizwal & \multirow{2}{*}{$\mathrm{MZ}$} & 23.73 & 92.72 & 950 & $(2001-2010)$ & NA & NA & NA & NA & NA & (1985-1991) \\
\hline 26 & Lengpui & & 23.83 & 92.62 & 432 & (2003-2010) & (2001-2010) & (2003-2010) & (2003-2010) & (2003-2010) & (2003-2010) & NA \\
\hline 27 & Dimapur & \multirow{2}{*}{ NL } & 25.83 & 93.75 & 268 & $(2000-2009)$ & NA & $(2000-2009)$ & $(2000-2009)$ & $(2000-2009)$ & & NA \\
\hline 28 & Kohima & & 25.63 & 94.17 & 1390 & (1971-2010) & (1971-2010) & (1971-2010) & (1971-2010) & (1971-2010) & (1971-1993) & (1971-1991) \\
\hline 29 & Agartala & \multirow{2}{*}{ TR } & 23.88 & 91.25 & 20 & (1971-1998) & (1971-2010) & (1971-2010) & (1971-2010) & (1971-2010) & (1971-2010) & (1971-1998) \\
\hline 30 & Kailashahar & & 24.32 & 92.00 & 30 & (1971-2010) & (1971-2010) & (1971-2010) & (1971-2010) & (1971-2010) & $(1971-2010)$ & (1971-1997) \\
\hline
\end{tabular}

*AP: Arunachal Pradesh, AS: Assam, MN: Manipur, ML: Meghalaya, MZ: Mizoram, NL: Nagaland, and TR: Tripura. 
where, $m$ is the number of tied groups and $t_{k}$ is the size of the $k^{\text {th }}$ tied group. Then, the standard normal test statistic $Z_{c}$ is given by:

$$
Z_{c}= \begin{cases}\frac{(S-1)}{\sigma_{S}}, & S>0 \\ 0, & S=0 \\ \frac{(S+1)}{\sigma_{s}}, & S<0\end{cases}
$$

The null hypothesis for "no trend" is rejected when:

$$
\left|Z_{c}\right|>Z_{1-\frac{\alpha}{2}}
$$

where, $Z$ is the standard normal variate and $\alpha$ is the level of significance (taken as $5 \%$ in this study).

To avoid falls positive MK test results due to presence of auto-correlation in the series, the effective sample size $n^{*}$ was calculated as (Matalas and Langbein, 1962):

$$
n^{*}=\frac{n}{1+2 \cdot \frac{\rho_{1}^{n+1}-n \cdot \rho_{1}^{2}+(n-1) \cdot \rho_{1}}{n \cdot\left(\rho_{1}-1\right)^{2}}}
$$

and the variance was modified as (Yue and Wang, 2004):

$$
\sigma_{s}^{*}{ }^{2}=\sigma_{S}^{2} \cdot \frac{n}{n^{*}}
$$

The $\rho_{1}$ in Eq. 6 is the lag- 1 auto-correlation coefficient determined as (Salas et al., 1980):

$$
\rho_{l}=\frac{\sum_{i=1}^{n-1}\left(x_{i}^{*}-\bar{x}^{*}\right)\left(x^{*}{ }_{i+1}-\bar{x}^{*}\right)}{\sum_{i=1}^{n}\left(x^{*}{ }_{i}-\bar{x}^{*}\right)^{2}}
$$

for the de-trended series $x^{*}{ }_{k}$ determined as:

$$
x_{k}^{*}=x_{k}-\beta \cdot(k-1), k=1,2, \ldots, n
$$

where $B$ is the Sen slope discussed below.

\subsubsection{Sen slope}

The magnitude of the trend is given as (Sen, 1968):

$$
\beta=\operatorname{Median}\left(\frac{x_{j}-x_{i}}{j-i}\right), \quad \forall i<j, \quad 1<i<j<n
$$

A positive (negative) value of $B$ indicates an increasing (decreasing) trend.

ArcTrends, a toolbar extension of ESRI ArcGIS developed by Bandyopadhyay et al. (2011), was used in this study for performing the above tests. This toolbar consists of four interactive tools (General Statistics, Trend Analysis, Delete Output Fields, and Change Projections). The General Statistics calculates the statistical parameters (minimum, maximum, mean, standard deviation, and coefficient of variation) of metrological data and helps in identifying the outliers. The Trend Analysis is for detection and quantification of trend in metrological data using MK test (with options to be selected for level of significance, consideration of auto-correlation, format of output, etc.) and/ or Sen slope.

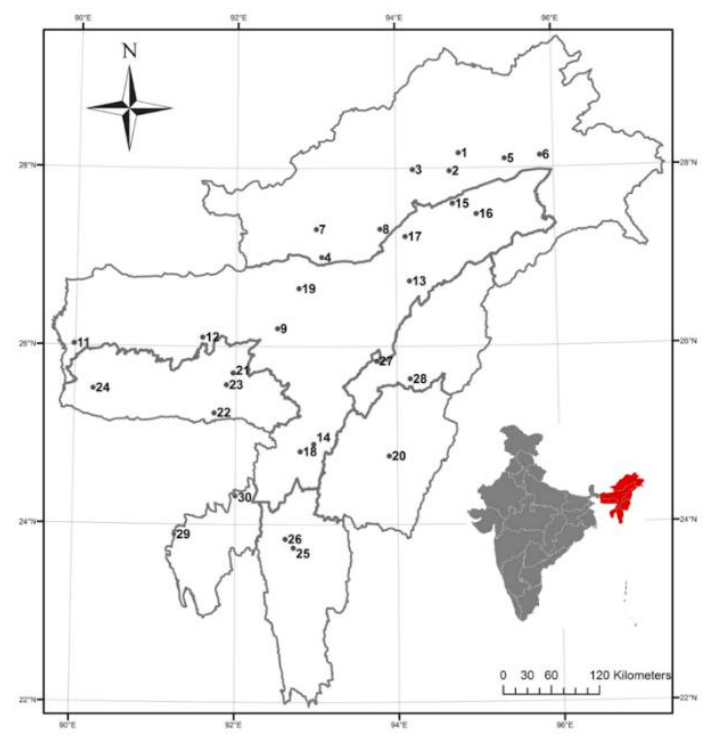

Figure 1. Map of NE India (excluding Sikkim) showing the location of the stations under study 2.4 The ArcTrends Toolbar

The outputs of the above two tools are written in the attribute table of the meteorological stations point coverage. The Delete Output Fields deletes all these output fields in a single click and readies the point coverage for new calculations. The Change Projection dropdown menu allows the user to display the map in either geographical or projected coordinate systems.

The time series data needs to be supplied to the toolbar as Comma Separated Values (CSV) in a CSV file containing two columns: date (as DD-MM-YYYY) and data, either in daily or monthly frequency. Any missing data are to be fed as "NA" or left blank against the date. These CSV files should be named as <station name>_<parameter name>.csv and the toolbar automatically reads the data corresponding to the stations selected.

The ArcTrends toolbar makes the user free from complicated interactions required for temporal trend estimation and incorporates all functions into a single toolbar. Since the results are updated automatically into the corresponding field of the attribute table of the selected layer, these can be directly used for visualization of the spatial pattern and further spatial analyses like spatial interpolation of $Z_{c}$ or Sens slope in the GIS environment.

\section{Results and discussion}

The MK test (5\% level of significance and considering autocorrelation) results and Sen slope values for various meteorological variables, namely, rainfall, number of rainy days, air temperature (maximum, minimum, and mean), mean relative humidity, and wind speed for different stations and states of NE India are presented in this section. MK test results are indicated by the symbols ' $Y+$ ' for significant positive, ' $Y$-' for significant negative, and ' $N$ ' for no significant trend. The Sen slope values are reported only for stations having significant trend. 
Table 2. Station-wise MK test results and Sen slopes $\left(\mathrm{mm}_{\mathrm{mear}} \mathrm{H}^{-1}\right)$ for monthly and yearly rainfall

\begin{tabular}{|c|c|c|c|c|c|c|c|c|c|c|c|c|c|c|c|}
\hline SI. No. & Station & State & Jan & Feb & Mar & Apr & May & Jun & Jul & Aug & Sep & Oct & Nov & Dec & Yearly \\
\hline & Aalo & \multirow{8}{*}{ AP } & $\mathrm{N}$ & $\mathrm{N}$ & $\mathrm{N}$ & $Y+(8.64)$ & $\mathrm{N}$ & $\mathrm{N}$ & $\mathrm{N}$ & $\mathrm{N}$ & $\mathrm{N}$ & $\mathrm{N}$ & $\mathrm{N}$ & $\mathrm{N}$ & $\mathrm{N}$ \\
\hline & Basar & & $\mathrm{N}$ & $\mathrm{N}$ & $\mathrm{N}$ & $\mathrm{N}$ & $\mathrm{N}$ & $\mathrm{N}$ & $\mathrm{N}$ & $\mathrm{N}$ & $\mathrm{N}$ & $\mathrm{N}$ & $\mathrm{N}$ & $\mathrm{N}$ & $\mathrm{N}$ \\
\hline & Daporijo & & $\mathrm{N}$ & $\mathrm{N}$ & $\mathrm{N}$ & $Y+(13.74)$ & $\mathrm{N}$ & $\mathrm{N}$ & $\mathrm{N}$ & $\mathrm{N}$ & $\mathrm{N}$ & $Y+(7.15)$ & $Y+(1.74)$ & $\mathrm{N}$ & $\mathrm{N}$ \\
\hline & Itanagar & & $\mathrm{N}$ & $\mathrm{N}$ & $\mathrm{N}$ & $\mathrm{N}$ & $\mathrm{N}$ & $\mathrm{N}$ & $\mathrm{N}$ & $\mathrm{N}$ & $\mathrm{N}$ & $N$ & $N$ & $\mathrm{~N}$ & $\mathrm{~N}$ \\
\hline & Passighat & & $\mathrm{N}$ & $\mathrm{N}$ & $\mathrm{N}$ & $\mathrm{N}$ & $\mathrm{N}$ & $\mathrm{N}$ & $\mathrm{N}$ & $\mathrm{N}$ & $\mathrm{N}$ & $\mathrm{N}$ & $\mathrm{N}$ & $\mathrm{N}$ & $\mathrm{N}$ \\
\hline & Roing & & $\mathrm{N}$ & $\mathrm{Y}+(17.41)$ & $\mathrm{N}$ & $\mathrm{N}$ & $\mathrm{N}$ & $\mathrm{N}$ & $\mathrm{N}$ & $\mathrm{N}$ & $\mathrm{N}$ & $Y-(-11.90)$ & $\mathrm{N}$ & $\mathrm{N}$ & $\mathrm{N}$ \\
\hline & Seppa & & $\mathrm{N}$ & $\mathrm{N}$ & $\mathrm{N}$ & $\mathrm{N}$ & $\mathrm{N}$ & $\mathrm{N}$ & $\mathrm{N}$ & $\mathrm{N}$ & $\mathrm{N}$ & $\mathrm{N}$ & $\mathrm{N}$ & $\mathrm{N}$ & $\mathrm{N}$ \\
\hline & Ziro & & $\mathrm{N}$ & $\mathrm{N}$ & $\mathrm{N}$ & $\mathrm{N}$ & $\mathrm{N}$ & $\mathrm{N}$ & Y- (-6.83) & Y- (-23.99) & $\mathrm{N}$ & $\mathrm{N}$ & $\mathrm{N}$ & $\mathrm{N}$ & $\mathrm{N}$ \\
\hline & Chaparmukh & \multirow{11}{*}{ AS } & $\mathrm{N}$ & $\mathrm{N}$ & $\mathrm{N}$ & $\mathrm{N}$ & $\mathrm{N}$ & $\mathrm{N}$ & $\mathrm{N}$ & $\mathrm{N}$ & $\mathrm{N}$ & $\mathrm{N}$ & $\mathrm{N}$ & $\mathrm{N}$ & $\mathrm{N}$ \\
\hline & Dibrugarh & & $\mathrm{N}$ & $\mathrm{N}$ & $Y+(2.36)$ & $\mathrm{N}$ & $\mathrm{N}$ & $\mathrm{N}$ & Y- (-4.03) & $\mathrm{N}$ & $\mathrm{N}$ & $\mathrm{N}$ & $\mathrm{N}$ & Y- $(-0.53)$ & $\mathrm{N}$ \\
\hline & Dhubri & & $\mathrm{N}$ & $\mathrm{N}$ & $\mathrm{N}$ & $\mathrm{N}$ & $\mathrm{N}$ & $\mathrm{N}$ & Y- $(-8.66)$ & $\mathrm{N}$ & $\mathrm{N}$ & $\mathrm{N}$ & $\mathrm{N}$ & $\mathrm{N}$ & $Y-(-1.63)$ \\
\hline & Guwahati & & $\mathrm{N}$ & $\mathrm{N}$ & $\mathrm{N}$ & $\mathrm{N}$ & $\mathrm{N}$ & $\mathrm{N}$ & $\mathrm{N}$ & $\mathrm{N}$ & $\mathrm{N}$ & $\mathrm{N}$ & $\mathrm{N}$ & $\mathrm{N}$ & $\mathrm{N}$ \\
\hline & Jorhat & & $\mathrm{N}$ & $\mathrm{N}$ & $\mathrm{N}$ & $\mathrm{N}$ & $\mathrm{N}$ & $\mathrm{N}$ & $\mathrm{N}$ & $\mathrm{N}$ & $\mathrm{N}$ & $\mathrm{N}$ & $\mathrm{N}$ & $\mathrm{N}$ & $\mathrm{N}$ \\
\hline & Kumbhigram & & $\mathrm{N}$ & $\mathrm{N}$ & $\mathrm{N}$ & $Y+(14.01)$ & $\mathrm{N}$ & $\mathrm{N}$ & $\mathrm{N}$ & $Y-(-8.42)$ & $\mathrm{N}$ & $\mathrm{N}$ & $\mathrm{N}$ & $\mathrm{N}$ & $\mathrm{N}$ \\
\hline & Likabali & & $\mathrm{N}$ & $\mathrm{N}$ & $\mathrm{N}$ & $\mathrm{N}$ & $\mathrm{N}$ & $\mathrm{N}$ & $\mathrm{N}$ & $\mathrm{N}$ & $\mathrm{N}$ & $\mathrm{N}$ & $\mathrm{N}$ & $\mathrm{N}$ & $\mathrm{N}$ \\
\hline & Mohanbari & & $\mathrm{N}$ & $\mathrm{N}$ & $\mathrm{N}$ & $\mathrm{N}$ & $\mathrm{N}$ & $Y+(18.1)$ & Y- (-19.33) & $\mathrm{N}$ & $\mathrm{N}$ & $\mathrm{N}$ & $\mathrm{N}$ & $\mathrm{N}$ & $\mathrm{N}$ \\
\hline & N. Lakhimpur & & $\mathrm{N}$ & $\mathrm{N}$ & $\mathrm{N}$ & $\mathrm{N}$ & $\mathrm{N}$ & $\mathrm{N}$ & $\mathrm{N}$ & $Y+(3.67)$ & $\mathrm{N}$ & $\mathrm{N}$ & $\mathrm{N}$ & $\mathrm{N}$ & $\mathrm{N}$ \\
\hline & Silchar & & $Y-(-0.30)$ & $\mathrm{N}$ & $\mathrm{N}$ & $\mathrm{N}$ & $\mathrm{N}$ & $\mathrm{N}$ & $\mathrm{N}$ & $\mathrm{N}$ & $\mathrm{N}$ & $\mathrm{N}$ & $\mathrm{N}$ & $\mathrm{N}$ & $\mathrm{N}$ \\
\hline & Tezpur & & $\mathrm{N}$ & $\mathrm{N}$ & $\mathrm{Y}+(0.77)$ & $\mathrm{Y}+(2.03)$ & $\mathrm{N}$ & $\mathrm{N}$ & $Y-(-4.45)$ & $\mathrm{N}$ & $\mathrm{N}$ & $\mathrm{N}$ & $\mathrm{N}$ & $\mathrm{N}$ & $\mathrm{N}$ \\
\hline & Imphal & $\mathrm{MN}$ & $\mathrm{N}$ & $\mathrm{N}$ & $\mathrm{N}$ & $\mathrm{N}$ & $\mathrm{Y}+(2.21)$ & $\mathrm{N}$ & $\mathrm{N}$ & $\mathrm{N}$ & $\mathrm{N}$ & $\mathrm{N}$ & $\mathrm{N}$ & $\mathrm{N}$ & $N$ \\
\hline & Barapani & \multirow{4}{*}{$\mathrm{ML}$} & $\mathrm{N}$ & $\mathrm{Y}-(-1.26)$ & $\mathrm{N}$ & $\mathrm{N}$ & $\mathrm{N}$ & $\mathrm{N}$ & $\mathrm{N}$ & $\mathrm{N}$ & $\mathrm{N}$ & $\mathrm{N}$ & $\mathrm{N}$ & $\mathrm{N}$ & $\mathrm{N}$ \\
\hline & Cherrapunji & & $\mathrm{N}$ & $\mathrm{N}$ & $\mathrm{N}$ & $\mathrm{N}$ & $\mathrm{N}$ & $\mathrm{N}$ & $\mathrm{Y}+(19.60)$ & $\mathrm{N}$ & $\mathrm{N}$ & $\mathrm{N}$ & $\mathrm{N}$ & $\mathrm{N}$ & $\mathrm{N}$ \\
\hline & Shillong & & $\mathrm{N}$ & $\mathrm{N}$ & $\mathrm{N}$ & $\mathrm{N}$ & $\mathrm{N}$ & $\mathrm{N}$ & $N$ & $\mathrm{~N}$ & $\mathrm{~N}$ & $\mathrm{~N}$ & $\mathrm{~N}$ & $\mathrm{~N}$ & $\mathrm{~N}$ \\
\hline & Tura & & $\mathrm{N}$ & $\mathrm{N}$ & $\mathrm{N}$ & $Y-(-12.12)$ & $\mathrm{N}$ & $\mathrm{N}$ & $\mathrm{N}$ & $\mathrm{N}$ & $\mathrm{N}$ & $\mathrm{N}$ & $\mathrm{N}$ & $\mathrm{N}$ & $\mathrm{N}$ \\
\hline & Aizawl & \multirow[b]{2}{*}{$\mathrm{MZ}$} & $\mathrm{N}$ & $\mathrm{N}$ & $\mathrm{N}$ & $\mathrm{N}$ & $Y-(-21.08)$ & $\mathrm{N}$ & $\mathrm{N}$ & $\mathrm{N}$ & $Y+(15.10)$ & $\mathrm{N}$ & $\mathrm{N}$ & $\mathrm{N}$ & $\mathrm{N}$ \\
\hline & Lengpui & & $\mathrm{N}$ & $\mathrm{N}$ & $\mathrm{N}$ & $\mathrm{N}$ & $\mathrm{N}$ & $\mathrm{N}$ & $\mathrm{N}$ & $\mathrm{N}$ & $\mathrm{N}$ & $Y+(13.27)$ & $Y+(1.02)$ & $\mathrm{N}$ & $\mathrm{N}$ \\
\hline & Dimapur & \multirow{2}{*}{ NL } & $\mathrm{N}$ & $\mathrm{N}$ & $\mathrm{N}$ & $\mathrm{N}$ & $\mathrm{N}$ & $\mathrm{N}$ & $\mathrm{Y}-(-16.9)$ & $\mathrm{Y}-(-22.17)$ & $\mathrm{N}$ & $\mathrm{N}$ & $\mathrm{N}$ & $\mathrm{N}$ & $\mathrm{N}$ \\
\hline & Kohima & & $\mathrm{N}$ & $\mathrm{N}$ & $\mathrm{N}$ & $\mathrm{N}$ & $\mathrm{N}$ & $\mathrm{N}$ & $\mathrm{N}$ & $\mathrm{N}$ & $\mathrm{N}$ & $\mathrm{N}$ & $\mathrm{N}$ & $\mathrm{N}$ & $\mathrm{N}$ \\
\hline & Agartala & \multirow{2}{*}{ TR } & $\mathrm{N}$ & $\mathrm{N}$ & $Y+(1.77)$ & $\mathrm{N}$ & $\mathrm{N}$ & $\mathrm{N}$ & $\mathrm{N}$ & $\mathrm{N}$ & $\mathrm{N}$ & $\mathrm{N}$ & $\mathrm{N}$ & $\mathrm{N}$ & $N$ \\
\hline & Kailashahar & & $\mathrm{N}$ & $\mathrm{N}$ & $\mathrm{N}$ & $\mathrm{N}$ & $\mathrm{N}$ & $\mathrm{N}$ & $\mathrm{N}$ & $\mathrm{N}$ & $\mathrm{N}$ & $\mathrm{N}$ & $Y-(-0.4)$ & $\mathrm{N}$ & $\mathrm{N}$ \\
\hline & No. of stations with $\mathrm{Y}_{+}$ & & 0 & 1 & 3 & 4 & 1 & 1 & 1 & 1 & 1 & 2 & 2 & 0 & 0 \\
\hline & No. of stations with Y- & & 1 & 1 & 0 & 1 & 1 & 0 & 6 & 3 & 0 & 1 & 1 & 1 & 1 \\
\hline
\end{tabular}

Table 3. State and region-wise MK test results and Sen slopes $\left(\mathrm{mm}\right.$ year $\left.{ }^{-1}\right)$ for monthly and yearly rainfall

\begin{tabular}{|c|c|c|c|c|c|c|c|c|c|c|c|c|c|}
\hline State & Jan & Feb & Mar & Apr & May & Jun & Jul & Aug & Sep & Oct & Nov & Dec & Yearly \\
\hline Arunachal Pradesh & $\mathrm{N}$ & $\mathrm{N}$ & $\mathrm{N}$ & $\mathrm{N}$ & $\mathrm{N}$ & $\mathrm{N}$ & $\mathrm{N}$ & $\mathrm{N}$ & $\mathrm{N}$ & $\mathrm{N}$ & $\mathrm{N}$ & $\mathrm{N}$ & $\mathrm{N}$ \\
\hline Assam & $\mathrm{N}$ & $\mathrm{N}$ & $\mathrm{Y}+(1.27)$ & $\mathrm{N}$ & $\mathrm{N}$ & N & $Y-(-4.49)$ & $\mathrm{N}$ & $\mathrm{N}$ & N & $N$ & $N$ & $Y-(-0.75)$ \\
\hline Manipur & $\mathrm{N}$ & $\mathrm{N}$ & $\mathrm{N}$ & $\mathrm{N}$ & $\mathrm{Y}+(2.21)$ & $\mathrm{N}$ & $\mathrm{N}$ & $\mathrm{N}$ & $\mathrm{N}$ & $\mathrm{N}$ & $\mathrm{N}$ & $\mathrm{N}$ & $\mathrm{N}$ \\
\hline Meghalaya & $\mathrm{N}$ & $\mathrm{N}$ & $\mathrm{N}$ & $\mathrm{N}$ & $\mathrm{N}$ & $\mathrm{N}$ & $\mathrm{N}$ & $\mathrm{N}$ & $\mathrm{N}$ & $\mathrm{N}$ & $\mathrm{N}$ & $\mathrm{N}$ & $\mathrm{N}$ \\
\hline Mizoram & $\mathrm{N}$ & $\mathrm{N}$ & $\mathrm{N}$ & $\mathrm{N}$ & $\mathrm{N}$ & N & N & $\mathrm{N}$ & $\mathrm{N}$ & $\mathrm{N}$ & $N$ & $N$ & $\mathrm{~N}$ \\
\hline Nagaland & $\mathrm{N}$ & $\mathrm{N}$ & N & $\mathrm{N}$ & $\mathrm{N}$ & N & N & $\mathrm{N}$ & $\mathrm{N}$ & $N$ & $N$ & $N$ & N \\
\hline Tripura & $\mathrm{N}$ & $\mathrm{N}$ & $Y+(0.84)$ & $\mathrm{N}$ & $\mathrm{N}$ & $\mathrm{N}$ & $\mathrm{N}$ & $\mathrm{N}$ & $\mathrm{N}$ & $\mathrm{N}$ & $\mathrm{N}$ & $\mathrm{N}$ & $\mathrm{N}$ \\
\hline NE India & $\mathrm{N}$ & $\mathrm{N}$ & $\mathrm{N}$ & $\mathrm{N}$ & $\mathrm{N}$ & $\mathrm{Y}-(-3.32)$ & $\mathrm{N}$ & $\mathrm{N}$ & $\mathrm{N}$ & $\mathrm{N}$ & $\mathrm{N}$ & $\mathrm{N}$ & $\mathrm{N}$ \\
\hline
\end{tabular}


Table 4. Station-wise MK test results and Sen slopes (year ${ }^{-1}$ ) for monthly and yearly no. of rainy days

\begin{tabular}{|c|c|c|c|c|c|c|c|c|c|c|c|c|c|c|c|}
\hline Sl. No. & Station & State & Jan & Feb & Mar & Apr & May & Jun & Jul & Aug & Sep & Oct & Nov & Dec & Yearly \\
\hline 1 & Aalo & \multirow{8}{*}{ AP } & - & - & - & - & - & - & - & - & - & - & - & - & - \\
\hline 2 & Basar & & - & - & - & - & - & - & - & - & - & - & - & - & - \\
\hline 3 & Daporijo & & - & - & - & - & - & - & - & - & - & - & - & - & - \\
\hline 4 & Itanagar & & $\mathrm{N}$ & $\mathrm{N}$ & $\mathrm{N}$ & $\mathrm{N}$ & $\mathrm{N}$ & $\mathrm{N}$ & $\mathrm{N}$ & $\mathrm{N}$ & $\mathrm{N}$ & $\mathrm{N}$ & $\mathrm{N}$ & $\mathrm{N}$ & $\mathrm{N}$ \\
\hline 5 & Passighat & & $\mathrm{N}$ & $\mathrm{N}$ & $\mathrm{N}$ & $\mathrm{N}$ & $\mathrm{N}$ & $\mathrm{N}$ & $\mathrm{N}$ & $\mathrm{N}$ & $\mathrm{N}$ & $\mathrm{N}$ & $\mathrm{N}$ & $\mathrm{N}$ & $\mathrm{N}$ \\
\hline 6 & Roing & & - & - & - & - & - & - & - & - & - & - & - & - & - \\
\hline 7 & Seppa & & $\mathrm{N}$ & $\mathrm{N}$ & $\mathrm{N}$ & $\mathrm{N}$ & $\mathrm{N}$ & $\mathrm{N}$ & $\mathrm{N}$ & $\mathrm{N}$ & $\mathrm{N}$ & $\mathrm{N}$ & $Y+(0.25)$ & $\mathrm{N}$ & $\mathrm{N}$ \\
\hline 8 & Ziro & & $\mathrm{N}$ & $\mathrm{N}$ & $\mathrm{N}$ & $\mathrm{N}$ & $\mathrm{N}$ & $\mathrm{N}$ & $\mathrm{N}$ & $\mathrm{N}$ & $\mathrm{N}$ & $\mathrm{N}$ & $\mathrm{N}$ & $\mathrm{N}$ & $\mathrm{N}$ \\
\hline 9 & Chaparmukh & \multirow{11}{*}{ AS } & $\mathrm{N}$ & $\mathrm{N}$ & $\mathrm{N}$ & $\mathrm{N}$ & $N$ & $\mathrm{~N}$ & $N$ & $\mathrm{~N}$ & $\mathrm{~N}$ & $\mathrm{~N}$ & $\mathrm{Y}-(-0.15)$ & $\mathrm{N}$ & $N$ \\
\hline 10 & Dibrugarh & & $\mathrm{N}$ & $\mathrm{N}$ & $Y+(0.17)$ & $\mathrm{N}$ & $\mathrm{N}$ & $\mathrm{N}$ & $\mathrm{N}$ & $\mathrm{N}$ & $\mathrm{N}$ & $\mathrm{N}$ & $N$ & $Y-(-0.05)$ & $\mathrm{N}$ \\
\hline 11 & Dhubri & & $\mathrm{N}$ & $\mathrm{N}$ & $N$ & $\mathrm{~N}$ & $\mathrm{~N}$ & $\mathrm{~N}$ & $\mathrm{~N}$ & $\mathrm{~N}$ & Y- $(-0.14)$ & $\mathrm{N}$ & $\mathrm{N}$ & $\mathrm{N}$ & $\mathrm{N}$ \\
\hline 12 & Guwahati & & $\mathrm{N}$ & $\mathrm{N}$ & $\mathrm{N}$ & $\mathrm{N}$ & $\mathrm{N}$ & $\mathrm{N}$ & $\mathrm{N}$ & $\mathrm{N}$ & $N$ & $\mathrm{~N}$ & $\mathrm{~N}$ & $\mathrm{~N}$ & $\mathrm{~N}$ \\
\hline 13 & Jorhat & & - & - & - & - & - & - & - & - & - & - & - & - & - \\
\hline 14 & Kumbhigram & & - & - & - & - & - & - & - & - & - & - & - & - & - \\
\hline 15 & Likabali & & - & - & - & - & - & - & - & - & - & - & - & - & - \\
\hline 16 & Mohanbari & & $\mathrm{N}$ & $\mathrm{N}$ & $\mathrm{N}$ & $\mathrm{N}$ & $\mathrm{N}$ & $\mathrm{N}$ & $\mathrm{N}$ & $\mathrm{N}$ & $\mathrm{N}$ & $\mathrm{N}$ & $\mathrm{N}$ & $\mathrm{N}$ & $\mathrm{N}$ \\
\hline 17 & N. Lakhimpur & & $\mathrm{N}$ & $\mathrm{N}$ & $Y+(0.10)$ & $\mathrm{N}$ & $\mathrm{N}$ & $\mathrm{Y}+(0.11)$ & $\mathrm{N}$ & $Y+(0.13)$ & $Y-(0.14)$ & $\mathrm{N}$ & $\mathrm{N}$ & $Y-(0.03)$ & $\mathrm{N}$ \\
\hline 18 & Silchar & & $\mathrm{N}$ & $\mathrm{N}$ & $N$ & $\mathrm{~N}$ & $Y+(0.21)$ & $N$ & $\mathrm{~N}$ & $\mathrm{~N}$ & $\mathrm{~N}$ & $\mathrm{~N}$ & $\mathrm{~N}$ & $\mathrm{~N}$ & $\mathrm{~N}$ \\
\hline 19 & Tezpur & & $\mathrm{N}$ & $\mathrm{N}$ & $Y+(0.09)$ & $\mathrm{Y}+(0.14)$ & $\mathrm{Y}+(0.11)$ & $\mathrm{N}$ & $\mathrm{N}$ & $\mathrm{N}$ & $\mathrm{N}$ & $\mathrm{N}$ & $\mathrm{N}$ & $\mathrm{N}$ & $\mathrm{N}$ \\
\hline 20 & Imphal & $\mathrm{MN}$ & $\mathrm{N}$ & $\mathrm{N}$ & $\mathrm{N}$ & $\mathrm{N}$ & $\mathrm{N}$ & $\mathrm{N}$ & $\mathrm{N}$ & $\mathrm{N}$ & $\mathrm{N}$ & $\mathrm{N}$ & $\mathrm{N}$ & $\mathrm{N}$ & $\mathrm{N}$ \\
\hline 21 & Barapani & \multirow{4}{*}{$\mathrm{ML}$} & $\mathrm{Y}-(-0.14)$ & Y- $(-0.18)$ & $\mathrm{N}$ & $\mathrm{N}$ & $N$ & $\mathrm{~N}$ & $\mathrm{~N}$ & $\mathrm{~N}$ & $\mathrm{~N}$ & $Y-(-0.20)$ & $\mathrm{N}$ & $\mathrm{N}$ & $\mathrm{N}$ \\
\hline 22 & Cherrapunji & & $\mathrm{N}$ & $\mathrm{N}$ & $\mathrm{N}$ & $\mathrm{N}$ & $\mathrm{N}$ & $\mathrm{N}$ & $\mathrm{N}$ & $\mathrm{N}$ & $\mathrm{N}$ & $\mathrm{N}$ & $\mathrm{N}$ & $\mathrm{N}$ & $\mathrm{N}$ \\
\hline 23 & Shillong & & $\mathrm{N}$ & $\mathrm{N}$ & $\mathrm{N}$ & $\mathrm{N}$ & $\mathrm{N}$ & $\mathrm{N}$ & $\mathrm{N}$ & $Y+(0.09)$ & $\mathrm{N}$ & $\mathrm{N}$ & $\mathrm{N}$ & $\mathrm{N}$ & $\mathrm{N}$ \\
\hline 24 & Tura & & $\mathrm{N}$ & $\mathrm{N}$ & $Y-(-0.25)$ & $Y-(-0.45)$ & $\mathrm{N}$ & $\mathrm{N}$ & $Y-(-0.70)$ & $\mathrm{N}$ & $\mathrm{N}$ & $\mathrm{N}$ & $\mathrm{N}$ & $\mathrm{N}$ & $\mathrm{N}$ \\
\hline 25 & Aizawl & \multirow{2}{*}{$\mathrm{MZ}$} & - & - & - & - & - & - & - & - & - & - & - & - & - \\
\hline 26 & Lengpui & & $\mathrm{N}$ & $\mathrm{N}$ & $\mathrm{N}$ & $\mathrm{N}$ & $\mathrm{N}$ & $\mathrm{N}$ & $\mathrm{N}$ & $\mathrm{N}$ & $\mathrm{N}$ & $\mathrm{N}$ & $\mathrm{N}$ & $\mathrm{N}$ & $\mathrm{N}$ \\
\hline 27 & Dimapur & \multirow{2}{*}{$\mathrm{NL}$} & - & - & - & - & - & - & - & - & - & - & - & - & - \\
\hline 28 & Kohima & & $\mathrm{N}$ & $\mathrm{N}$ & $\mathrm{N}$ & $\mathrm{N}$ & $\mathrm{N}$ & $\mathrm{N}$ & $\mathrm{N}$ & $\mathrm{N}$ & $\mathrm{N}$ & $\mathrm{N}$ & $\mathrm{N}$ & $\mathrm{N}$ & $\mathrm{N}$ \\
\hline 29 & Agartala & \multirow{2}{*}{ TR } & $\mathrm{N}$ & $\mathrm{N}$ & $\mathrm{N}$ & $\mathrm{N}$ & $\mathrm{N}$ & $\mathrm{N}$ & $\mathrm{N}$ & $\mathrm{N}$ & $\mathrm{N}$ & $\mathrm{N}$ & $\mathrm{Y}-(-0.04)$ & $\mathrm{N}$ & $\mathrm{N}$ \\
\hline 30 & Kailashahar & & $\mathrm{N}$ & $\mathrm{N}$ & $Y+(0.08)$ & $Y+(0.27)$ & $Y+(0.32)$ & $Y+(0.36)$ & $\mathrm{N}$ & $\mathrm{N}$ & $Y+(0.09)$ & $\mathrm{N}$ & $\mathrm{N}$ & $\mathrm{N}$ & $Y+(0.1)$ \\
\hline \multirow{2}{*}{\multicolumn{2}{|c|}{$\begin{array}{l}\text { No. of stations with } \mathrm{Y}_{+} \\
\text {No. of stations with } \mathrm{Y} \text { - }\end{array}$}} & & 0 & 0 & 4 & 2 & 3 & 2 & 0 & 2 & 1 & 0 & 1 & 0 & 1 \\
\hline & & & 1 & 1 & 1 & 1 & 0 & 0 & 1 & 0 & 2 & 1 & 2 & 2 & 0 \\
\hline
\end{tabular}

Table 5. State and region-wise MK test results and Sen slopes (year $\left.{ }^{-1}\right)$ for monthly and yearly no. of rainy days

\begin{tabular}{|c|c|c|c|c|c|c|c|c|c|c|c|c|c|}
\hline State & Jan & Feb & Mar & Apr & May & Jun & Jul & Aug & Sep & Oct & Nov & Dec & Yearly \\
\hline Arunachal Pradesh & $\mathrm{N}$ & $\mathrm{N}$ & $\mathrm{N}$ & $\mathrm{N}$ & $\mathrm{N}$ & $\mathrm{N}$ & $\mathrm{N}$ & $\mathrm{N}$ & $\mathrm{N}$ & $\mathrm{N}$ & $\mathrm{N}$ & $\mathrm{N}$ & $\mathrm{N}$ \\
\hline Assam & $\mathrm{N}$ & $\mathrm{N}$ & $Y+(0.09)$ & $\mathrm{N}$ & $\mathrm{N}$ & $Y+(0.10)$ & $\mathrm{N}$ & $Y+(0.09)$ & $\mathrm{N}$ & $\mathrm{N}$ & $\mathrm{N}$ & $Y-(-0.02)$ & $Y+(0.04)$ \\
\hline Manipur & $\mathrm{N}$ & $\mathrm{N}$ & $N$ & $\mathrm{~N}$ & $\mathrm{~N}$ & $\mathrm{~N}$ & $\mathrm{~N}$ & $\mathrm{~N}$ & $\mathrm{~N}$ & $\mathrm{~N}$ & $\mathrm{~N}$ & $\mathrm{~N}$ & $\mathrm{~N}$ \\
\hline Meghalaya & $\mathrm{N}$ & $\mathrm{N}$ & $\mathrm{N}$ & $\mathrm{N}$ & $\mathrm{N}$ & $\mathrm{N}$ & $\mathrm{N}$ & $\mathrm{N}$ & $\mathrm{N}$ & $\mathrm{N}$ & $\mathrm{N}$ & $\mathrm{N}$ & $\mathrm{N}$ \\
\hline Mizoram & $\mathrm{N}$ & $\mathrm{N}$ & $\mathrm{N}$ & $\mathrm{N}$ & $\mathrm{N}$ & $\mathrm{N}$ & $\mathrm{N}$ & $\mathrm{N}$ & $\mathrm{N}$ & $\mathrm{N}$ & $\mathrm{N}$ & $\mathrm{N}$ & $\mathrm{N}$ \\
\hline Nagaland & $\mathrm{N}$ & $\mathrm{N}$ & $\mathrm{N}$ & $\mathrm{N}$ & $\mathrm{N}$ & $\mathrm{N}$ & $\mathrm{N}$ & $\mathrm{N}$ & $\mathrm{N}$ & $\mathrm{N}$ & $\mathrm{N}$ & $\mathrm{N}$ & $\mathrm{N}$ \\
\hline Tripura & $\mathrm{N}$ & $\mathrm{N}$ & $\mathrm{N}$ & $Y+(0.11)$ & $Y+(0.14)$ & $Y+(0.12)$ & $\mathrm{N}$ & $\mathrm{N}$ & $Y+(0.08)$ & $Y+(0.05)$ & $\mathrm{N}$ & $\mathrm{N}$ & $Y+(0.05)$ \\
\hline NE India & $\mathrm{N}$ & $\mathrm{N}$ & $Y+(0.05)$ & $\mathrm{N}$ & $\mathrm{N}$ & $Y+(0.10)$ & $\mathrm{N}$ & $Y+(0.06)$ & $\mathrm{N}$ & $\mathrm{N}$ & $\mathrm{N}$ & $\mathrm{N}$ & $Y+(0.03)$ \\
\hline
\end{tabular}


Table 6. Station-wise MK test results and Sen slopes $\left({ }^{\circ} \mathrm{C}\right.$ year $\left.{ }^{-1}\right)$ for monthly and yearly average maximum temperature

\begin{tabular}{|c|c|c|c|c|c|c|c|c|c|c|c|c|c|c|c|}
\hline Sl. No. & Station & State & Jan & Feb & Mar & Apr & May & Jun & Jul & Aug & Sep & Oct & Nov & Dec & Yearly \\
\hline 1 & Aalo & \multirow{8}{*}{$\mathrm{AP}$} & $\mathrm{N}$ & $\mathrm{N}$ & $\mathrm{N}$ & $\mathrm{N}$ & $\mathrm{N}$ & $\mathrm{N}$ & $\mathrm{N}$ & $\mathrm{N}$ & $\mathrm{N}$ & $\mathrm{N}$ & $\mathrm{N}$ & $Y+(0.57)$ & $Y+(0.28)$ \\
\hline 2 & Basar & & $\mathrm{N}$ & $\mathrm{N}$ & $\mathrm{N}$ & $\mathrm{N}$ & $\mathrm{N}$ & $\mathrm{N}$ & $\mathrm{N}$ & $\mathrm{N}$ & $\mathrm{N}$ & $\mathrm{N}$ & $\mathrm{N}$ & $\mathrm{N}$ & $\mathrm{N}$ \\
\hline 3 & Daporijo & & - & - & - & - & - & - & - & - & - & - & - & - & - \\
\hline 4 & Itanagar & & $\mathrm{N}$ & $\mathrm{N}$ & $\mathrm{N}$ & $\mathrm{N}$ & $\mathrm{N}$ & $\mathrm{N}$ & $\mathrm{N}$ & $\mathrm{N}$ & $\mathrm{N}$ & $\mathrm{N}$ & $\mathrm{N}$ & $\mathrm{N}$ & $\mathrm{N}$ \\
\hline 5 & Passighat & & $\mathrm{N}$ & $\mathrm{N}$ & $Y-(-0.05)$ & $Y-(-0.04)$ & $\mathrm{N}$ & $\mathrm{N}$ & $\mathrm{N}$ & $\mathrm{N}$ & $\mathrm{N}$ & $\mathrm{N}$ & $\mathrm{N}$ & $Y+(0.03)$ & $\mathrm{N}$ \\
\hline 6 & Roing & & $\mathrm{N}$ & $Y+(1.00)$ & $N$ & $N$ & $\mathrm{~N}$ & $\mathrm{~N}$ & $\mathrm{~N}$ & $\mathrm{~N}$ & $\mathrm{~N}$ & $\mathrm{~N}$ & $\mathrm{~N}$ & $\mathrm{~N}$ & $\mathrm{~N}$ \\
\hline 7 & Seppa & & $\mathrm{N}$ & $\mathrm{N}$ & $\mathrm{N}$ & $\mathrm{N}$ & $\mathrm{N}$ & $\mathrm{N}$ & $\mathrm{N}$ & $\mathrm{N}$ & $\mathrm{N}$ & $\mathrm{N}$ & $\mathrm{N}$ & $\mathrm{N}$ & $\mathrm{N}$ \\
\hline 8 & Ziro & & $\mathrm{N}$ & $\mathrm{N}$ & $\mathrm{N}$ & $\mathrm{N}$ & $\mathrm{N}$ & $\mathrm{N}$ & $\mathrm{N}$ & $\mathrm{N}$ & $\mathrm{N}$ & $\mathrm{N}$ & $\mathrm{N}$ & $\mathrm{N}$ & $\mathrm{N}$ \\
\hline 9 & Chaparmukh & \multirow{11}{*}{ AS } & $N$ & $N$ & $N$ & $N$ & $\mathrm{~N}$ & $\mathrm{~N}$ & $\mathrm{~N}$ & $\mathrm{~N}$ & $\mathrm{~N}$ & $\mathrm{~N}$ & $\mathrm{~N}$ & $\mathrm{~N}$ & $N$ \\
\hline 10 & Dibrugarh & & $\mathrm{N}$ & $\mathrm{N}$ & $Y-(-0.10)$ & $\mathrm{N}$ & $\mathrm{N}$ & $\mathrm{N}$ & $\mathrm{N}$ & $\mathrm{N}$ & $\mathrm{N}$ & $\mathrm{N}$ & $Y+(0.06)$ & $Y+(0.08)$ & $\mathrm{N}$ \\
\hline 11 & Dhubri & & $Y-(-0.07)$ & $Y-(-0.05)$ & $Y-(-0.06)$ & $\mathrm{N}$ & $\mathrm{N}$ & $\mathrm{N}$ & $\mathrm{N}$ & $\mathrm{N}$ & $\mathrm{N}$ & $Y-(-0.05)$ & $\mathrm{N}$ & $Y-(-0.05)$ & $Y-(-0.03)$ \\
\hline 12 & Guwahati & & $\mathrm{N}$ & $\mathrm{N}$ & $\mathrm{N}$ & $\mathrm{N}$ & $\mathrm{Y}+(0.04)$ & $Y+(0.02)$ & $Y+(0.03)$ & $Y+(0.04)$ & $Y+(0.03)$ & $\mathrm{N}$ & $Y+(0.04)$ & $Y+(0.04)$ & $\mathrm{N}$ \\
\hline 13 & Jorhat & & $\mathrm{N}$ & $\mathrm{N}$ & $\mathrm{N}$ & $\mathrm{N}$ & $Y-(-0.72)$ & $\mathrm{N}$ & $\mathrm{N}$ & $\mathrm{N}$ & $\mathrm{N}$ & $\mathrm{N}$ & $\mathrm{N}$ & $\mathrm{N}$ & $\mathrm{N}$ \\
\hline 14 & Kumbhigram & & $\mathrm{N}$ & $\mathrm{N}$ & $\mathrm{N}$ & $\mathrm{N}$ & $N$ & $Y+(0.15)$ & $\mathrm{N}$ & $Y+(0.09)$ & $\mathrm{N}$ & $\mathrm{N}$ & $\mathrm{N}$ & $\mathrm{N}$ & $N$ \\
\hline 15 & Likabali & & $\mathrm{N}$ & $\mathrm{N}$ & $\mathrm{N}$ & $\mathrm{N}$ & $\mathrm{N}$ & $\mathrm{N}$ & $\mathrm{N}$ & $\mathrm{N}$ & $\mathrm{N}$ & $\mathrm{N}$ & $\mathrm{N}$ & $\mathrm{N}$ & $\mathrm{N}$ \\
\hline 16 & Mohanbari & & $\mathrm{N}$ & $\mathrm{N}$ & $\mathrm{N}$ & $\mathrm{N}$ & $\mathrm{N}$ & $\mathrm{N}$ & $\mathrm{N}$ & $\mathrm{N}$ & $\mathrm{N}$ & $\mathrm{N}$ & $\mathrm{N}$ & $\mathrm{N}$ & $\mathrm{N}$ \\
\hline 17 & N. Lakhimpur & & $Y+(0.05)$ & $Y+(0.05)$ & $\mathrm{N}$ & $\mathrm{N}$ & $Y+(0.04)$ & $\mathrm{N}$ & $Y+(0.03)$ & $\mathrm{N}$ & $Y+(0.05)$ & $Y+(0.05)$ & $Y+(0.05)$ & $Y+(0.07)$ & $Y+(0.04)$ \\
\hline 18 & Silchar & & $\mathrm{N}$ & $\mathrm{N}$ & $Y+(0.11)$ & $\mathrm{N}$ & $\mathrm{N}$ & $\mathrm{N}$ & $Y+(0.07)$ & $\mathrm{N}$ & $N$ & $\mathrm{~N}$ & $\mathrm{~N}$ & $\mathrm{~N}$ & $Y+(0.04)$ \\
\hline 19 & Tezpur & & $\mathrm{N}$ & $\mathrm{N}$ & $\mathrm{N}$ & $\mathrm{N}$ & $\mathrm{N}$ & $\mathrm{N}$ & $\mathrm{N}$ & $\mathrm{N}$ & $\mathrm{N}$ & $\mathrm{N}$ & $\mathrm{N}$ & $\mathrm{N}$ & $\mathrm{N}$ \\
\hline 20 & Imphal & $\mathrm{MN}$ & $Y+(0.06)$ & $\mathrm{Y}+(0.07)$ & $\mathrm{N}$ & $\mathrm{N}$ & $\mathrm{N}$ & $Y+(0.03)$ & $\mathrm{Y}+(0.03)$ & $\mathrm{Y}+(0.04)$ & $\mathrm{Y}+(0.05)$ & $Y+(0.03)$ & $Y+(0.04)$ & $\mathrm{Y}+(0.04)$ & $Y+(0.04)$ \\
\hline 21 & Barapani & \multirow{4}{*}{$M L$} & $Y+(0.12)$ & $N$ & $Y+(0.07)$ & $\mathrm{N}$ & $N$ & $\mathrm{~N}$ & $Y+(0.04)$ & $Y+(0.06)$ & $Y+(0.05)$ & $Y+(0.05)$ & $Y+(0.05)$ & $\mathrm{N}$ & $\mathrm{N}$ \\
\hline 22 & Cherrapunji & & $Y+(0.05)$ & $\mathrm{N}$ & $\mathrm{N}$ & $Y+(0.03)$ & $\mathrm{Y}+(0.04)$ & $\mathrm{N}$ & $\mathrm{N}$ & $Y+(0.03)$ & $Y+(0.04)$ & $Y+(0.03)$ & $Y+(0.04)$ & $Y+(0.05)$ & $Y+(0.03)$ \\
\hline 23 & Shillong & & $Y+(0.03)$ & $\mathrm{N}$ & $\mathrm{N}$ & $\mathrm{N}$ & $\mathrm{N}$ & $Y+(0.02)$ & $\mathrm{N}$ & $Y+(0.02)$ & $Y+(0.02)$ & $\mathrm{N}$ & $\mathrm{N}$ & $\mathrm{N}$ & $Y+(0.01)$ \\
\hline 24 & Tura & & $Y-(-0.32)$ & $Y-(-0.42)$ & Y- $(0.45)$ & $\mathrm{N}$ & $\mathrm{N}$ & $\mathrm{N}$ & $\mathrm{N}$ & $\mathrm{N}$ & $\mathrm{N}$ & $\mathrm{N}$ & $\mathrm{N}$ & $Y-(-0.43)$ & $Y-(0.21)$ \\
\hline 25 & Aizawl & \multirow{2}{*}{$\mathrm{MZ}$} & - & - & - & - & - & - & - & - & - & - & - & - & - \\
\hline 26 & Lengpui & & $Y+(0.44)$ & $\mathrm{N}$ & $Y+(0.38)$ & $\mathrm{Y}+(0.33)$ & $\mathrm{N}$ & $\mathrm{N}$ & $\mathrm{N}$ & $\mathrm{N}$ & $\mathrm{N}$ & $Y+(0.06)$ & $\mathrm{N}$ & $\mathrm{N}$ & $\mathrm{N}$ \\
\hline 27 & Dimapur & \multirow{2}{*}{$\mathrm{NL}$} & $\mathrm{N}$ & $\mathrm{N}$ & $N$ & $N$ & $\mathrm{Y}+(0.36)$ & $Y+(0.13)$ & $N$ & $Y+(0.22)$ & $Y+(0.22)$ & $\mathrm{N}$ & $Y+(0.17)$ & $\mathrm{N}$ & $N$ \\
\hline 28 & Kohima & & $\mathrm{N}$ & $\mathrm{N}$ & $\mathrm{N}$ & $\mathrm{N}$ & $N$ & $\mathrm{~N}$ & $\mathrm{~N}$ & $\mathrm{~N}$ & $\mathrm{~N}$ & $\mathrm{~N}$ & $\mathrm{~N}$ & $\mathrm{~N}$ & $\mathrm{~N}$ \\
\hline 29 & Agartala & \multirow{2}{*}{ TR } & $\mathrm{Y}-(-0.03)$ & $\mathrm{N}$ & $\mathrm{N}$ & $\mathrm{N}$ & $\mathrm{Y}+(0.04)$ & $Y+(0.03)$ & $\mathrm{Y}+(0.03)$ & $Y+(0.04)$ & $Y+(0.03)$ & $\mathrm{N}$ & $\mathrm{N}$ & $\mathrm{N}$ & $\mathrm{N}$ \\
\hline 30 & Kailashahar & & $\mathrm{N}$ & $\mathrm{N}$ & $\mathrm{N}$ & $\mathrm{N}$ & $\mathrm{N}$ & $\mathrm{N}$ & $\mathrm{N}$ & $Y+(0.02)$ & $\mathrm{N}$ & $\mathrm{N}$ & $Y+(0.02)$ & $Y+(0.02)$ & $Y+(0.01)$ \\
\hline \multirow{2}{*}{\multicolumn{2}{|c|}{$\begin{array}{l}\text { No. of stations with Y+ } \\
\text { No. of stations with Y- }\end{array}$}} & & 6 & 3 & 3 & 2 & 5 & 6 & 6 & 9 & 8 & 5 & 8 & 8 & 7 \\
\hline & & & 3 & 2 & 4 & 1 & 1 & 0 & 0 & 0 & 0 & 1 & 0 & 2 & 2 \\
\hline
\end{tabular}




\subsection{Precipitation}

\subsubsection{Rainfall}

Table 2 displays the results of the MK test in the monthly and yearly total rainfall data, along with the corresponding Sen slope values for statistically significant trends. March and April had the highest number of stations (three and four, respectively) with significant positive trend, followed by October and November with two stations each. On the other hand, July had the highest number (six) of stations with significant negative trend followed by August with three stations. In all the months, majority of the stations didn't show any significant trend. In case of yearly rainfall, all the stations reported no significant trend except Dhubri. Cherrapunji reported the highest increasing slope of 19.60 $\mathrm{mm}$ per year in July, whereas, Ziro showed the steepest negative slope of $-23.99 \mathrm{~mm}$ per year in August.

When assessed state-wise averages (Table 3), Assam and Tripura showed significant positive trend in March with slope of 1.27 and $0.84 \mathrm{~mm}$ per year, respectively, while Manipur showed it in May with Sen slope of $2.21 \mathrm{~mm}$ per year. Negative trend was found only in Assam in July and in yearly data with slopes of -4.49 and $-0.75 \mathrm{~mm}$ per year, respectively. Average rainfall over entire NE India reported a significant decreasing trend in June (-3.32 $\mathrm{mm}$ per year) only.

\subsubsection{Number of rainy days}

Table 4 shows the results of the MK test in the monthly and yearly total number of rainy days of 21 stations, along with the Sen slope values corresponding to statistically significant trends, as data were not available for nine stations (Aalo, Basar, Daporijo, Roing, Jorhat, Kumbhigram, Likabali, Aizawl, and Dimapur). A large number of stations didn't show any significant trend in any of the months. March had the highest number (four) of stations with significant positive trend, followed by May with three and April, June and August with two stations each. Very few stations reported significant negative trend, the highest being two stations each in September, November and December. Kailashahar reported the highest increasing slope of 0.36 day per year in June, whereas, Tura showed the highest negative slope of -0.70 day per year in July.

In case of state averages (Table 5), Assam and Tripura had presence of significant positive trend in a few months during March-October, as well as in the yearly total. The highest positive Sen slope (0.14 day per year) was found in Tripura in May, whereas, negative trend was found only in Assam with a slope of -0.02 day per year in December. When considered the entire region as a whole, March, June, August, and the yearly total reported significant increasing trend.

\subsection{Temperature}

\subsubsection{Maximum temperature}

Table 6 shows the results of the MK test in the monthly and yearly average maximum temperature of 28 stations, along with the Sen slope values corresponding to significant trends. Maximum temperature data of two stations (Aizwal and Daporijo) were not available. August had maximum (nine) stations with significant rising trend, followed by September, November and December with eight stations each; January, June and July with six stations each; May and October with five stations each; February and March with three stations each; and April with only two stations. On the other hand, March had maximum (four) stations with significant falling trend, followed by January with three stations, December with two stations, and April, May and October with only one station each. No stations reported any significant falling trend during monsoon months (JuneSeptember) and November. In the yearly average data, seven stations were found to have significant rising trend, whereas, two stations reported significant falling trend.

Table 7 presents the state and region-average results. Manipur reported a warming trend almost throughout the year barring three months before monsoon (March-May). Only Assam reported a statistically significant falling trend, that too only in two months (March and April), and no trend was found in the other months. All other states reported rising trend in maximum temperature over onefive months in the year. Significant rising trend was found during July-September $\left(0.03^{\circ} \mathrm{C}\right.$ per year) when the entire region was considered as a whole.

\subsubsection{Minimum temperature}

Table 8 shows the MK test results on minimum temperature for 27 stations, along with the Sen slopes associated with significant positive or negative trends. Minimum temperature data were not available for Aizwal, Roing and Daporijo stations. The yearly average minimum temperature tested positive for statistically significant rising trend in 11 stations. The monthly tests identified minimum five to maximum nine stations with significant increasing trend in all the months. On the contrary, number of stations with significant decreasing trend was comparatively less and ranged between zero and three. Two stations in Assam, Dhubri and Guwahati, reported significant rising trend in all the months, closely followed by Seppa in Arunachal Pradesh and Kailashahar in Tripura with 11 months each.

In case of state averages (Table 9), Arunachal Pradesh didn't report any significant trend in any of the months, whereas, Tripura was found to have significant increasing trend in nine months followed by Manipur in five months. Significant negative trend was seen only in Meghalaya for three months (February, March and November). Assam, Manipur and Tripura reported significant increasing trend in the yearly average minimum temperature. The NE regional average showed significant rising trend during May-October and December.

\subsubsection{Mean temperature}

Table 10 displays the MK test results on mean temperature for 27 stations, along with the Sen slope values for significant trend cases. 
Table 7. State and region-wise MK test results and Sen slopes $\left({ }^{\circ} \mathrm{C}\right.$ year $\left.{ }^{-1}\right)$ for monthly and yearly average maximum temperature

\begin{tabular}{|c|c|c|c|c|c|c|c|c|c|c|c|c|c|}
\hline State & Jan & Feb & Mar & Apr & May & Jun & Jul & Aug & Sep & Oct & Nov & Dec & Yearly \\
\hline Arunachal Pradesh & $N$ & $\mathrm{~N}$ & $\mathrm{~N}$ & $\mathrm{~N}$ & $\mathrm{~N}$ & $\mathrm{~N}$ & $Y+(0.07)$ & $\mathrm{N}$ & $\mathrm{Y}+(0.10)$ & $\mathrm{N}$ & $\mathrm{N}$ & $\mathrm{N}$ & $\mathrm{N}$ \\
\hline Assam & $\mathrm{N}$ & $\mathrm{N}$ & $Y-(-0.06)$ & $Y-(-0.04)$ & $\mathrm{N}$ & $\mathrm{N}$ & $\mathrm{N}$ & $\mathrm{N}$ & $\mathrm{N}$ & $\mathrm{N}$ & $\mathrm{N}$ & $\mathrm{N}$ & $\mathrm{N}$ \\
\hline Manipur & $Y+(0.06)$ & $Y+(0.07)$ & $\mathrm{N}$ & $\mathrm{N}$ & $\mathrm{N}$ & $Y+(0.03)$ & $Y+(0.03)$ & $Y+(0.04)$ & $Y+(0.05)$ & $Y+(0.03)$ & $Y+(0.04)$ & $Y+(0.04)$ & $Y+(0.04)$ \\
\hline Meghalaya & $\mathrm{N}$ & $\mathrm{N}$ & $\mathrm{N}$ & $\mathrm{N}$ & $\mathrm{N}$ & $\mathrm{N}$ & $\mathrm{N}$ & $Y+(0.04)$ & $\mathrm{N}$ & $\mathrm{N}$ & $\mathrm{N}$ & $N$ & $\mathrm{~N}$ \\
\hline Mizoram & $Y+(0.44)$ & $\mathrm{N}$ & $\mathrm{Y}+(0.38)$ & $Y+(0.33)$ & $\mathrm{N}$ & $\mathrm{N}$ & $\mathrm{N}$ & $\mathrm{N}$ & $\mathrm{N}$ & $Y+(0.06)$ & $\mathrm{N}$ & $\mathrm{N}$ & $\mathrm{N}$ \\
\hline Nagaland & $\mathrm{N}$ & $\mathrm{N}$ & $Y+(0.11)$ & $Y+(0.12)$ & $Y+(0.18)$ & $\mathrm{N}$ & $\mathrm{N}$ & $\mathrm{N}$ & $\mathrm{N}$ & $\mathrm{N}$ & $\mathrm{N}$ & $\mathrm{N}$ & $\mathrm{N}$ \\
\hline Tripura & $\mathrm{N}$ & $\mathrm{N}$ & $\mathrm{N}$ & $\mathrm{N}$ & $\mathrm{N}$ & $\mathrm{Y}+(0.02)$ & $Y+(0.02)$ & $Y+(0.03)$ & $Y+(0.03)$ & $Y+(0.01)$ & $\mathrm{N}$ & $\mathrm{N}$ & $Y+(0.01)$ \\
\hline NE India & $\mathrm{N}$ & $\mathrm{N}$ & $\mathrm{N}$ & $\mathrm{N}$ & $\mathrm{N}$ & $\mathrm{N}$ & $Y+(0.03)$ & $Y+(0.03)$ & $Y+(0.03)$ & $\mathrm{N}$ & $\mathrm{N}$ & $\mathrm{N}$ & $\mathrm{N}$ \\
\hline
\end{tabular}

Table 8. Station-wise MK test results and Sen slopes $\left({ }^{\circ} \mathrm{C}\right.$ year $\left.{ }^{-1}\right)$ for monthly and yearly average minimum temperature

\begin{tabular}{|c|c|c|c|c|c|c|c|c|c|c|c|c|c|c|c|}
\hline Sl. No. & Station & State & Jan & Feb & Mar & Apr & May & Jun & Jul & Aug & Sep & Oct & Nov & Dec & Yearly \\
\hline 1 & Aalo & \multirow{8}{*}{ AP } & $\mathrm{Y}-(-0.16)$ & $\mathrm{N}$ & $\mathrm{N}$ & $\mathrm{N}$ & $\mathrm{N}$ & $\mathrm{N}$ & $Y-(-0.15)$ & $\mathrm{N}$ & $\mathrm{N}$ & $Y-(-0.25)$ & $\mathrm{N}$ & $Y-(0.29)$ & $Y-(0.15)$ \\
\hline 2 & Basar & & $\mathrm{N}$ & $\mathrm{N}$ & $\mathrm{N}$ & $Y-(-0.24)$ & $\mathrm{N}$ & $\mathrm{N}$ & $Y-(-0.12)$ & $\mathrm{N}$ & $\mathrm{N}$ & $\mathrm{N}$ & $\mathrm{N}$ & $\mathrm{N}$ & $\mathrm{N}$ \\
\hline 3 & Daporijo & & - & - & - & - & - & - & - & - & - & - & - & - & - \\
\hline 4 & Itanagar & & $\mathrm{N}$ & $\mathrm{N}$ & $Y+(0.25)$ & $\mathrm{N}$ & $Y+(0.17)$ & $\mathrm{Y}+(0.24)$ & $\mathrm{N}$ & $Y+(0.18)$ & $\mathrm{N}$ & $\mathrm{N}$ & $\mathrm{N}$ & $Y+(0.30)$ & $Y+(0.13)$ \\
\hline 5 & Passighat & & $Y+(0.02)$ & $\mathrm{N}$ & $\mathrm{N}$ & $\mathrm{N}$ & $\mathrm{N}$ & $\mathrm{N}$ & $\mathrm{N}$ & $\mathrm{N}$ & $\mathrm{N}$ & $\mathrm{N}$ & $\mathrm{N}$ & $\mathrm{N}$ & $Y+(0.01)$ \\
\hline 6 & Roing & & - & - & - & - & - & - & - & - & - & - & - & - & - \\
\hline 7 & Seppa & & $Y+(0.61)$ & $Y+(0.71)$ & $Y+(0.66)$ & $\mathrm{Y}+(0.80)$ & $\mathrm{N}$ & $\mathrm{Y}+(0.31)$ & $Y+(0.40)$ & $Y+(0.40)$ & $Y+(0.47)$ & $Y+(0.62)$ & $Y+(0.52)$ & $Y+(0.49)$ & $Y+(0.50)$ \\
\hline 8 & Ziro & & $\mathrm{N}$ & $\mathrm{N}$ & $\mathrm{N}$ & $\mathrm{N}$ & $\mathrm{N}$ & $\mathrm{N}$ & $\mathrm{N}$ & $\mathrm{N}$ & $\mathrm{N}$ & $\mathrm{N}$ & $\mathrm{N}$ & $\mathrm{N}$ & $\mathrm{N}$ \\
\hline 9 & Chaparmukh & \multirow{11}{*}{ AS } & $\mathrm{N}$ & $\mathrm{N}$ & $\mathrm{N}$ & $\mathrm{N}$ & $\mathrm{N}$ & $\mathrm{N}$ & $\mathrm{N}$ & $\mathrm{N}$ & $\mathrm{N}$ & $\mathrm{N}$ & $\mathrm{N}$ & $N$ & $\mathrm{~N}$ \\
\hline 10 & Dibrugarh & & $\mathrm{N}$ & $Y+(0.05)$ & $Y+(0.04)$ & $\mathrm{N}$ & $\mathrm{N}$ & $\mathrm{N}$ & $\mathrm{Y}+(0.02)$ & $Y+(0.02)$ & $Y+(0.02)$ & $\mathrm{N}$ & $Y+(0.08)$ & $Y+(0.06)$ & $Y+(0.04)$ \\
\hline 11 & Dhubri & & $Y+(0.12)$ & $Y+(0.15)$ & $Y+(0.12)$ & $\mathrm{Y}+(0.04)$ & $Y+(0.04)$ & $Y+(0.03)$ & $Y+(0.04)$ & $Y+(0.05)$ & $Y+(0.06)$ & $Y+(0.06)$ & $Y+(0.10)$ & $Y+(0.14)$ & $Y+(0.08)$ \\
\hline 12 & Guwahati & & $Y+(0.04)$ & $Y+(0.06)$ & $Y+(0.03)$ & $\mathrm{Y}+(0.03)$ & $Y+(0.03)$ & $Y+(0.03)$ & $Y+(0.04)$ & $Y+(0.04)$ & $Y+(0.04)$ & $Y+(0.03)$ & $Y+(0.03)$ & $Y+(0.05)$ & $Y+(0.04)$ \\
\hline 13 & Jorhat & & $\mathrm{N}$ & $\mathrm{N}$ & $\mathrm{N}$ & $\mathrm{N}$ & $Y-(-0.35)$ & $\mathrm{N}$ & $\mathrm{N}$ & $\mathrm{N}$ & $\mathrm{N}$ & $\mathrm{N}$ & $\mathrm{N}$ & $\mathrm{N}$ & $\mathrm{N}$ \\
\hline 14 & Kumbhigram & & $\mathrm{N}$ & $\mathrm{N}$ & $\mathrm{N}$ & $\mathrm{N}$ & $N$ & $\mathrm{~N}$ & $\mathrm{~N}$ & $\mathrm{~N}$ & $\mathrm{~N}$ & $\mathrm{~N}$ & $\mathrm{~N}$ & $\mathrm{~N}$ & $\mathrm{~N}$ \\
\hline 15 & Likabali & & $Y-(-0.21)$ & $\mathrm{N}$ & $\mathrm{N}$ & $Y-(-0.21)$ & $\mathrm{N}$ & $\mathrm{N}$ & $\mathrm{N}$ & $\mathrm{N}$ & $\mathrm{N}$ & $\mathrm{N}$ & $\mathrm{N}$ & $\mathrm{N}$ & $\mathrm{N}$ \\
\hline 16 & Mohanbari & & $Y+(0.22)$ & $\mathrm{N}$ & $\mathrm{N}$ & $N$ & $\mathrm{~N}$ & $\mathrm{~N}$ & $\mathrm{~N}$ & $\mathrm{~N}$ & $\mathrm{~N}$ & $Y+(0.13)$ & $\mathrm{N}$ & $\mathrm{N}$ & $\mathrm{N}$ \\
\hline 17 & N. Lakhimpur & & $\mathrm{N}$ & $\mathrm{N}$ & $\mathrm{N}$ & $\mathrm{N}$ & $\mathrm{N}$ & $\mathrm{N}$ & $\mathrm{N}$ & $\mathrm{N}$ & $\mathrm{N}$ & $\mathrm{N}$ & $\mathrm{N}$ & $\mathrm{N}$ & $\mathrm{N}$ \\
\hline 18 & Silchar & & $\mathrm{N}$ & $\mathrm{N}$ & $\mathrm{N}$ & $\mathrm{Y}+(0.07)$ & $\mathrm{N}$ & $\mathrm{N}$ & $\mathrm{N}$ & $\mathrm{N}$ & $\mathrm{N}$ & $\mathrm{N}$ & $\mathrm{N}$ & $\mathrm{N}$ & $\mathrm{N}$ \\
\hline 19 & Tezpur & & $\mathrm{N}$ & $\mathrm{N}$ & $\mathrm{N}$ & $\mathrm{N}$ & $\mathrm{N}$ & $\mathrm{N}$ & $\mathrm{N}$ & $\mathrm{N}$ & $\mathrm{N}$ & $\mathrm{N}$ & $\mathrm{N}$ & $\mathrm{N}$ & $\mathrm{N}$ \\
\hline 20 & Imphal & $\mathrm{MN}$ & $\mathrm{N}$ & $\mathrm{N}$ & $Y+(0.05)$ & $\mathrm{N}$ & $\mathrm{N}$ & $\mathrm{N}$ & $\mathrm{Y}+(0.03)$ & $Y+(0.03)$ & $Y+(0.04)$ & $\mathrm{N}$ & $\mathrm{N}$ & $Y+(0.06)$ & $Y+(0.04)$ \\
\hline 21 & Barapani & \multirow{4}{*}{$\mathrm{ML}$} & $\mathrm{N}$ & $\mathrm{Y}+(0.10)$ & $N$ & $Y+0.05)$ & $\mathrm{N}$ & $\mathrm{N}$ & $\mathrm{N}$ & $\mathrm{N}$ & $\mathrm{N}$ & $\mathrm{N}$ & $\mathrm{N}$ & $Y+(0.10)$ & $Y+(0.05)$ \\
\hline 22 & Cherrapunji & & $\mathrm{N}$ & $\mathrm{N}$ & $\mathrm{N}$ & $N$ & $Y+(0.03)$ & $\mathrm{N}$ & $\mathrm{N}$ & $\mathrm{N}$ & $\mathrm{N}$ & $\mathrm{N}$ & $\mathrm{N}$ & $\mathrm{N}$ & $N$ \\
\hline 23 & Shillong & & $\mathrm{N}$ & $\mathrm{N}$ & $\mathrm{N}$ & $\mathrm{N}$ & $\mathrm{N}$ & $\mathrm{N}$ & $\mathrm{N}$ & $\mathrm{N}$ & $\mathrm{N}$ & $\mathrm{N}$ & $\mathrm{N}$ & $\mathrm{N}$ & $Y+(0.01)$ \\
\hline 24 & Tura & & $\mathrm{N}$ & $\mathrm{N}$ & $\mathrm{N}$ & $\mathrm{N}$ & $\mathrm{N}$ & $\mathrm{N}$ & $\mathrm{N}$ & $\mathrm{N}$ & $\mathrm{N}$ & $\mathrm{N}$ & $\mathrm{N}$ & $\mathrm{N}$ & $\mathrm{N}$ \\
\hline 25 & Aizawl & \multirow{2}{*}{ MZ } & - & - & - & - & - & - & - & - & - & - & - & - & - \\
\hline 26 & Lengpui & & $\mathrm{N}$ & $\mathrm{N}$ & $\mathrm{N}$ & $\mathrm{N}$ & $\mathrm{N}$ & $\mathrm{N}$ & $\mathrm{N}$ & $\mathrm{N}$ & $\mathrm{N}$ & $\mathrm{N}$ & $Y+(0.30)$ & $\mathrm{N}$ & $\mathrm{N}$ \\
\hline 27 & Dimapur & \multirow{2}{*}{ NL } & $\mathrm{N}$ & $\mathrm{N}$ & $\mathrm{N}$ & Y- $(-0.20)$ & $\mathrm{Y}-(-0.30)$ & $\mathrm{Y}-(0.30)$ & $\mathrm{Y}-(-0.10)$ & $\mathrm{Y}-(-0.15)$ & $\mathrm{Y}-(-0.22)$ & $\mathrm{N}$ & $\mathrm{Y}-(-0.22)$ & $\mathrm{N}$ & $\mathrm{N}$ \\
\hline 28 & Kohima & & $\mathrm{N}$ & $\mathrm{N}$ & $\mathrm{N}$ & $\mathrm{N}$ & $\mathrm{N}$ & $\mathrm{N}$ & $\mathrm{N}$ & $\mathrm{N}$ & $\mathrm{N}$ & $\mathrm{N}$ & $\mathrm{N}$ & $\mathrm{N}$ & $\mathrm{N}$ \\
\hline 29 & Agartala & \multirow{2}{*}{$\mathrm{TR}$} & $Y+(0.06)$ & $Y+(0.04)$ & $N$ & $N$ & $Y+(0.03)$ & $Y+(0.02)$ & $\mathrm{Y}+(0.02)$ & $Y+(0.02)$ & $Y+(0.03)$ & $\mathrm{N}$ & $\mathrm{N}$ & $Y+(0.08)$ & $Y+(0.03)$ \\
\hline 30 & Kailashahar & & $Y+(0.06)$ & $Y+(0.05)$ & $Y+(0.06)$ & $\mathrm{Y}+(0.03)$ & $Y+(0.05)$ & $\mathrm{N}$ & $Y+(0.03)$ & $Y+(0.03)$ & $Y+(0.03)$ & $Y+(0.03)$ & $Y+(0.05)$ & $Y+(0.08)$ & $Y+(0.04)$ \\
\hline \multirow{2}{*}{\multicolumn{2}{|c|}{$\begin{array}{l}\text { No. of stations with Y+ } \\
\text { No. of stations with Y- }\end{array}$}} & & 7 & 7 & 7 & 6 & 6 & 5 & 7 & 8 & 7 & 5 & 6 & 9 & 11 \\
\hline & & & 2 & 0 & 0 & 3 & 2 & 1 & 3 & 1 & 1 & 1 & 1 & 1 & 1 \\
\hline
\end{tabular}


Table 9. State and region-wise MK test results and Sen slopes $\left({ }^{\circ} \mathrm{C}\right.$ year $\left.{ }^{-1}\right)$ for monthly and yearly average minimum temperature

\begin{tabular}{|c|c|c|c|c|c|c|c|c|c|c|c|c|c|}
\hline State & Jan & Feb & Mar & Apr & May & Jun & Jul & Aug & Sep & Oct & Nov & Dec & Yearly \\
\hline Arunachal Pradesh & $\mathrm{N}$ & $\mathrm{N}$ & $\mathrm{N}$ & $\mathrm{N}$ & $\mathrm{N}$ & $\mathrm{N}$ & $\mathrm{N}$ & $\mathrm{N}$ & $\mathrm{N}$ & $\mathrm{N}$ & $\mathrm{N}$ & $\mathrm{N}$ & $\mathrm{N}$ \\
\hline Assam & $\mathrm{N}$ & $\mathrm{N}$ & $\mathrm{N}$ & $\mathrm{N}$ & $\mathrm{N}$ & $\mathrm{N}$ & $\mathrm{N}$ & $\mathrm{N}$ & $Y+(0.02)$ & $\mathrm{N}$ & $\mathrm{N}$ & $Y+(0.04)$ & $Y+(0.02)$ \\
\hline Manipur & $\mathrm{N}$ & $\mathrm{N}$ & $Y+(0.05)$ & $\mathrm{N}$ & $\mathrm{N}$ & $\mathrm{N}$ & $Y+(0.03)$ & $\mathrm{Y}+(0.03)$ & $Y+(0.04)$ & $\mathrm{N}$ & $\mathrm{N}$ & $Y+(0.06)$ & $Y+(0.04)$ \\
\hline Meghalaya & $\mathrm{N}$ & $Y-(-0.04)$ & $Y-(-0.06)$ & $\mathrm{N}$ & $\mathrm{N}$ & $\mathrm{N}$ & $\mathrm{N}$ & $\mathrm{N}$ & $\mathrm{N}$ & $\mathrm{N}$ & $Y-(-0.03)$ & $\mathrm{N}$ & $\mathrm{N}$ \\
\hline Mizoram & $\mathrm{N}$ & $\mathrm{N}$ & $\mathrm{N}$ & $\mathrm{N}$ & $\mathrm{N}$ & $\mathrm{N}$ & $\mathrm{N}$ & $\mathrm{N}$ & $\mathrm{N}$ & $\mathrm{N}$ & $Y+(0.30)$ & $\mathrm{N}$ & $\mathrm{N}$ \\
\hline Nagaland & $\mathrm{N}$ & $\mathrm{N}$ & $\mathrm{N}$ & $\mathrm{N}$ & $\mathrm{N}$ & $\mathrm{N}$ & $Y+(0.11)$ & $\mathrm{N}$ & $\mathrm{N}$ & $\mathrm{N}$ & $\mathrm{N}$ & $\mathrm{N}$ & $\mathrm{N}$ \\
\hline Tripura & $\mathrm{Y}+(0.06)$ & $Y+(0.05)$ & $\mathrm{N}$ & $\mathrm{N}$ & $Y+(0.03)$ & $Y+(0.02)$ & $Y+(0.02)$ & $Y+(0.03)$ & $Y+(0.03)$ & $Y+(0.04)$ & $\mathrm{N}$ & $Y+(0.08)$ & $Y+(0.04)$ \\
\hline NE India & $\mathrm{N}$ & $\mathrm{N}$ & $\mathrm{N}$ & $\mathrm{N}$ & $Y+(0.03)$ & $Y+(0.02)$ & $Y+(0.03)$ & $Y+(0.03)$ & $Y+(0.03)$ & $Y+(0.02)$ & $\mathrm{N}$ & $Y+(0.04)$ & $Y+(0.02)$ \\
\hline
\end{tabular}

Table 10. Station-wise MK test results and Sen slopes $\left({ }^{\circ} \mathrm{C}\right.$ year $\left.{ }^{-1}\right)$ for monthly and yearly average mean temperature

\begin{tabular}{|c|c|c|c|c|c|c|c|c|c|c|c|c|c|c|c|}
\hline SI. No. & Station & State & Jan & Feb & Mar & Apr & May & Jun & Jul & Aug & Sep & Oct & Nov & Dec & Yearly \\
\hline 1 & Aalo & \multirow{8}{*}{ AP } & $\mathrm{N}$ & $\mathrm{N}$ & $\mathrm{N}$ & $\mathrm{N}$ & $\mathrm{N}$ & $\mathrm{N}$ & $\mathrm{N}$ & $\mathrm{N}$ & $\mathrm{N}$ & $\mathrm{N}$ & $\mathrm{N}$ & $\mathrm{N}$ & $\mathrm{N}$ \\
\hline 2 & Basar & & $\mathrm{N}$ & $\mathrm{N}$ & $\mathrm{N}$ & $\mathrm{N}$ & $\mathrm{N}$ & $\mathrm{N}$ & $\mathrm{N}$ & $\mathrm{N}$ & $\mathrm{N}$ & $\mathrm{N}$ & $\mathrm{N}$ & $\mathrm{N}$ & $\mathrm{N}$ \\
\hline 3 & Daporijo & & - & - & - & - & - & - & - & - & - & - & - & - & - \\
\hline 4 & Itanagar & & $\mathrm{N}$ & $\mathrm{N}$ & $\mathrm{N}$ & $\mathrm{N}$ & $\mathrm{N}$ & $\mathrm{N}$ & $\mathrm{N}$ & $Y+(0.13)$ & $\mathrm{N}$ & $\mathrm{N}$ & $\mathrm{N}$ & $\mathrm{N}$ & $\mathrm{N}$ \\
\hline 5 & Passighat & & $\mathrm{N}$ & $\mathrm{N}$ & $\mathrm{N}$ & $\mathrm{N}$ & $\mathrm{N}$ & $\mathrm{N}$ & $\mathrm{N}$ & $N$ & $\mathrm{~N}$ & $\mathrm{~N}$ & $\mathrm{~N}$ & $Y+(0.03)$ & $\mathrm{N}$ \\
\hline 6 & Roing & & - & - & - & - & - & - & - & - & - & - & - & - & - \\
\hline 7 & Seppa & & $Y+(0.26)$ & $\mathrm{N}$ & $Y+(0.18)$ & $\mathrm{N}$ & $\mathrm{N}$ & $\mathrm{N}$ & $\mathrm{N}$ & $Y+(0.15)$ & $Y+(0.16)$ & $Y+(0.24)$ & $\mathrm{N}$ & $Y+(0.18)$ & $\mathrm{Y}+(0.13)$ \\
\hline 8 & Ziro & & $\mathrm{N}$ & $\mathrm{N}$ & $\mathrm{N}$ & $\mathrm{N}$ & $\mathrm{N}$ & $\mathrm{N}$ & $\mathrm{N}$ & $\mathrm{N}$ & $\mathrm{N}$ & $\mathrm{N}$ & $\mathrm{N}$ & $\mathrm{N}$ & $\mathrm{N}$ \\
\hline 9 & Chaparmukh & \multirow{11}{*}{ AS } & $\mathrm{N}$ & $\mathrm{Y}-(-0.17)$ & $\mathrm{N}$ & $\mathrm{N}$ & $\mathrm{N}$ & $\mathrm{N}$ & $\mathrm{N}$ & $\mathrm{N}$ & $\mathrm{N}$ & $\mathrm{N}$ & $\mathrm{N}$ & $\mathrm{N}$ & $\mathrm{N}$ \\
\hline 10 & Dibrugarh & & $\mathrm{N}$ & $\mathrm{N}$ & $\mathrm{N}$ & $\mathrm{N}$ & $\mathrm{N}$ & $\mathrm{N}$ & $\mathrm{N}$ & $\mathrm{N}$ & $\mathrm{N}$ & $\mathrm{Y}+(0.03)$ & $Y+(0.07)$ & $Y+(0.07)$ & $\mathrm{Y}+(0.02)$ \\
\hline 11 & Dhubri & & $Y+(0.03)$ & $Y+(0.05)$ & $\mathrm{N}$ & $\mathrm{N}$ & $\mathrm{N}$ & $\mathrm{N}$ & $Y+(0.02)$ & $Y+(0.03)$ & $Y+(0.02)$ & $\mathrm{N}$ & $Y+(0.03)$ & $Y+(0.05)$ & $Y+(0.02)$ \\
\hline 12 & Guwahati & & $\mathrm{N}$ & $Y+(0.04)$ & $\mathrm{N}$ & $\mathrm{N}$ & $Y+(0.04)$ & $Y+(0.03)$ & $Y+(0.04)$ & $Y+(0.04)$ & $Y+(0.04)$ & $Y+(0.03)$ & $Y+(0.04)$ & $Y+(0.05)$ & $\mathrm{Y}+(0.03)$ \\
\hline 13 & Jorhat & & $\mathrm{N}$ & $\mathrm{N}$ & $\mathrm{N}$ & $\mathrm{N}$ & $\mathrm{N}$ & $\mathrm{N}$ & $\mathrm{N}$ & $\mathrm{N}$ & $\mathrm{N}$ & $\mathrm{N}$ & $\mathrm{N}$ & $\mathrm{N}$ & $\mathrm{N}$ \\
\hline 14 & Kumbhigram & & N & $\mathrm{N}$ & $\mathrm{N}$ & $\mathrm{N}$ & $\mathrm{N}$ & $\mathrm{Y}+(0.11)$ & N & $Y+(0.06)$ & N & $\mathrm{N}$ & $\mathrm{N}$ & N & $\mathrm{N}$ \\
\hline 15 & Likabali & & $\mathrm{N}$ & $\mathrm{N}$ & $\mathrm{N}$ & $\mathrm{N}$ & $\mathrm{N}$ & $\mathrm{N}$ & $\mathrm{N}$ & $\mathrm{N}$ & $\mathrm{N}$ & $\mathrm{N}$ & $\mathrm{N}$ & $\mathrm{N}$ & $\mathrm{N}$ \\
\hline 16 & Mohanbari & & $Y+(0.13)$ & $\mathrm{N}$ & $\mathrm{N}$ & $\mathrm{N}$ & $\mathrm{N}$ & $\mathrm{N}$ & $\mathrm{N}$ & $\mathrm{N}$ & $\mathrm{N}$ & $\mathrm{Y}+(0.14)$ & $\mathrm{N}$ & $\mathrm{N}$ & $\mathrm{N}$ \\
\hline 17 & N. Lakhimpur & & $Y+(0.03)$ & $Y+(0.04)$ & $\mathrm{N}$ & $\mathrm{N}$ & $Y+(0.02)$ & $\mathrm{N}$ & $\mathrm{N}$ & $\mathrm{N}$ & $Y+(0.03)$ & $Y+(0.03)$ & $Y+(0.03)$ & $Y+(0.05)$ & $\mathrm{Y}+(0.02)$ \\
\hline 18 & Silchar & & $\mathrm{N}$ & $\mathrm{N}$ & $Y+(0.08)$ & $\mathrm{N}$ & $N$ & $\mathrm{~N}$ & $Y+(0.07)$ & $\mathrm{N}$ & $Y+(0.05)$ & $Y+(0.05)$ & $Y-(-0.05)$ & $\mathrm{N}$ & $Y+(0.05)$ \\
\hline 19 & Tezpur & & $\mathrm{N}$ & $\mathrm{N}$ & $\mathrm{N}$ & $\mathrm{N}$ & $\mathrm{N}$ & $\mathrm{N}$ & $\mathrm{N}$ & $\mathrm{N}$ & $\mathrm{N}$ & $\mathrm{N}$ & $\mathrm{N}$ & $\mathrm{N}$ & $\mathrm{N}$ \\
\hline 20 & Imphal & $\mathrm{MN}$ & $\mathrm{Y}+(0.04)$ & $\mathrm{Y}+(0.05)$ & $Y+(0.04)$ & $Y+(0.03)$ & $Y+(0.04)$ & $\mathrm{Y}+(0.02)$ & $Y+(0.04)$ & $Y+(0.04)$ & $Y+(0.05)$ & $Y+(0.04)$ & $\mathrm{Y}+(0.03)$ & $Y+(0.05)$ & $\mathrm{Y}+(0.04)$ \\
\hline 21 & Barapani & \multirow{4}{*}{ ML } & $Y+(0.08)$ & $\mathrm{N}$ & $\mathrm{N}$ & $\mathrm{N}$ & $N$ & $N$ & $Y+(0.04)$ & $Y+(0.05)$ & $\mathrm{N}$ & $\mathrm{Y}+(0.04)$ & $\mathrm{N}$ & $Y+(0.08)$ & $Y+(0.05)$ \\
\hline 22 & Cherrapunji & & $\mathrm{N}$ & $\mathrm{N}$ & $\mathrm{N}$ & $\mathrm{N}$ & $Y+(0.03)$ & $\mathrm{N}$ & $\mathrm{N}$ & $Y+(0.02)$ & $Y+(0.03)$ & $\mathrm{N}$ & $\mathrm{N}$ & $\mathrm{N}$ & $\mathrm{N}$ \\
\hline 23 & Shillong & & $\mathrm{N}$ & $\mathrm{N}$ & $\mathrm{N}$ & $\mathrm{N}$ & $Y+(0.02)$ & $\mathrm{N}$ & $\mathrm{N}$ & $Y+(0.01)$ & $Y+(0.02)$ & $\mathrm{N}$ & $\mathrm{N}$ & $\mathrm{N}$ & $\mathrm{Y}+(0.01)$ \\
\hline 24 & Tura & & $\mathrm{N}$ & $\mathrm{N}$ & Y- $(-0.34)$ & $\mathrm{N}$ & $Y-(-0.27)$ & $\mathrm{N}$ & $\mathrm{N}$ & $\mathrm{N}$ & $\mathrm{N}$ & $\mathrm{N}$ & $\mathrm{N}$ & $\mathrm{N}$ & $\mathrm{N}$ \\
\hline 25 & Aizawl & \multirow{2}{*}{$\mathrm{MZ}$} & - & - & - & - & - & - & - & - & - & - & - & - & - \\
\hline 26 & Lengpui & & $\mathrm{N}$ & $\mathrm{N}$ & $\mathrm{N}$ & $\mathrm{N}$ & $\mathrm{N}$ & $\mathrm{N}$ & $\mathrm{N}$ & $\mathrm{N}$ & $\mathrm{N}$ & $\mathrm{N}$ & $\mathrm{N}$ & $\mathrm{N}$ & $\mathrm{N}$ \\
\hline 27 & Dimapur & \multirow{2}{*}{ NL } & $\mathrm{N}$ & $\mathrm{N}$ & $\mathrm{N}$ & $\mathrm{N}$ & $\mathrm{N}$ & $\mathrm{N}$ & $\mathrm{N}$ & $\mathrm{N}$ & $\mathrm{N}$ & $\mathrm{N}$ & $\mathrm{N}$ & $\mathrm{N}$ & $\mathrm{N}$ \\
\hline 28 & Kohima & & $\mathrm{N}$ & $\mathrm{N}$ & $\mathrm{N}$ & $\mathrm{N}$ & $\mathrm{N}$ & $\mathrm{N}$ & $\mathrm{N}$ & $\mathrm{N}$ & $\mathrm{N}$ & $\mathrm{N}$ & $\mathrm{N}$ & $Y-(0.08)$ & $\mathrm{N}$ \\
\hline 29 & Agartala & \multirow{2}{*}{$\mathrm{TR}$} & $\mathrm{N}$ & $\mathrm{Y}+(0.04)$ & $\mathrm{N}$ & $\mathrm{N}$ & $Y+(0.04)$ & $\mathrm{Y}+(0.03)$ & $Y+(0.02)$ & $Y+(0.03)$ & $Y+(0.03)$ & $Y+(0.02)$ & $\mathrm{N}$ & $\mathrm{Y}+(0.05)$ & $\mathrm{Y}+(0.02)$ \\
\hline 30 & Kailashahar & & $Y+(0.03)$ & $Y+(0.03)$ & $Y+(0.03)$ & $\mathrm{N}$ & $Y+(0.04)$ & $N$ & $Y+(0.02)$ & $Y+(0.02)$ & $Y+(0.02)$ & $Y+(0.02)$ & $Y+(0.03)$ & $Y+(0.05)$ & $Y+(0.03)$ \\
\hline \multirow{2}{*}{\multicolumn{2}{|c|}{$\begin{array}{l}\text { No. of stations with } \mathrm{Y}+ \\
\text { No. of stations with } \mathrm{Y} \text { - }\end{array}$}} & & 7 & 6 & 4 & 1 & 7 & 4 & 7 & 11 & 10 & 10 & 6 & 10 & 11 \\
\hline & & & 0 & 1 & 1 & 0 & 1 & 0 & 0 & 0 & 0 & 0 & 1 & 1 & 0 \\
\hline
\end{tabular}


Table 11. State and region-wise MK test results and Sen slopes $\left({ }^{\circ} \mathrm{C}\right.$ year $\left.{ }^{-1}\right)$ for monthly and yearly average mean temperature

\begin{tabular}{|c|c|c|c|c|c|c|c|c|c|c|c|c|c|}
\hline State & Jan & Feb & Mar & Apr & May & Jun & Jul & Aug & Sep & Oct & Nov & Dec & Yearly \\
\hline Arunachal Pradesh & $\mathrm{N}$ & $\mathrm{N}$ & $\mathrm{N}$ & $\mathrm{N}$ & $\mathrm{N}$ & $\mathrm{N}$ & $Y+(0.07)$ & $\mathrm{N}$ & $\mathrm{Y}+(0.10)$ & $\mathrm{N}$ & $\mathrm{N}$ & $\mathrm{N}$ & $\mathrm{N}$ \\
\hline Assam & $\mathrm{N}$ & $\mathrm{N}$ & $\mathrm{N}$ & $\mathrm{N}$ & $\mathrm{N}$ & $\mathrm{N}$ & $\mathrm{N}$ & $\mathrm{N}$ & $\mathrm{N}$ & $\mathrm{N}$ & $\mathrm{N}$ & $Y+(0.02)$ & $\mathrm{N}$ \\
\hline Manipur & $Y+(0.04)$ & $Y+(0.05)$ & $\mathrm{Y}+(0.04)$ & $Y+(0.03)$ & $Y+(0.04)$ & $Y+(0.02)$ & $Y+(0.04)$ & $\mathrm{Y}+(0.04)$ & $Y+(0.05)$ & $Y+(0.04)$ & $\mathrm{Y}+(0.03)$ & $Y+(0.05)$ & $Y+(0.04)$ \\
\hline Meghalaya & $\mathrm{N}$ & $\mathrm{N}$ & $\mathrm{N}$ & $\mathrm{N}$ & $\mathrm{N}$ & $\mathrm{N}$ & $\mathrm{N}$ & $Y+(0.03)$ & $\mathrm{N}$ & $\mathrm{N}$ & $\mathrm{N}$ & $\mathrm{N}$ & $\mathrm{N}$ \\
\hline Mizoram & $\mathrm{N}$ & $\mathrm{N}$ & $\mathrm{N}$ & $\mathrm{N}$ & $\mathrm{N}$ & $\mathrm{N}$ & $\mathrm{N}$ & $\mathrm{N}$ & $\mathrm{N}$ & $\mathrm{N}$ & $\mathrm{N}$ & $\mathrm{N}$ & $\mathrm{N}$ \\
\hline Nagaland & $\mathrm{N}$ & $\mathrm{N}$ & $\mathrm{N}$ & $Y+(0.12)$ & $Y+(0.04)$ & $\mathrm{N}$ & $Y+(0.12)$ & $\mathrm{N}$ & $\mathrm{N}$ & $\mathrm{N}$ & $\mathrm{N}$ & $\mathrm{N}$ & $\mathrm{N}$ \\
\hline Tripura & $Y+(0.02)$ & $Y+(0.04)$ & $\mathrm{N}$ & $\mathrm{N}$ & $Y+(0.04)$ & $\mathrm{N}$ & $Y+(0.02)$ & $\mathrm{Y}+(0.03)$ & $Y+(0.03)$ & $\mathrm{Y}+(0.03)$ & $\mathrm{Y}+(0.03)$ & $Y+(0.05)$ & $Y+(0.03)$ \\
\hline NE India & $\mathrm{N}$ & $\mathrm{N}$ & $\mathrm{N}$ & $\mathrm{N}$ & $Y+(0.02)$ & $\mathrm{N}$ & $Y+(0.03)$ & $\mathrm{Y}+(0.03)$ & $\mathrm{Y}+(0.04)$ & $\mathrm{N}$ & $\mathrm{N}$ & $\mathrm{Y}+(0.03)$ & $Y+(0.02)$ \\
\hline
\end{tabular}

Table 12. Station-wise MK test results and Sen slopes $\left(\%\right.$ year $\left.{ }^{-1}\right)$ for monthly and yearly average relative humidity

\begin{tabular}{|c|c|c|c|c|c|c|c|c|c|c|c|c|c|c|c|}
\hline SI. No. & Station & State & Jan & Feb & Mar & Apr & May & Jun & Jul & Aug & Sep & Oct & Nov & Dec & Yearly \\
\hline 1 & Aalo & \multirow{8}{*}{$A P$} & $\mathrm{~N}$ & $\mathrm{~N}$ & $\mathrm{~N}$ & $\mathrm{~N}$ & $\mathrm{~N}$ & $\mathrm{~N}$ & $\mathrm{~N}$ & $\mathrm{~N}$ & $\mathrm{~N}$ & $\mathrm{~N}$ & $\mathrm{~N}$ & $\mathrm{~N}$ & $\mathrm{~N}$ \\
\hline 2 & Basar & & $\mathrm{N}$ & $\mathrm{N}$ & $\mathrm{N}$ & $\mathrm{N}$ & $\mathrm{N}$ & $\mathrm{N}$ & $\mathrm{N}$ & $\mathrm{N}$ & $\mathrm{N}$ & $\mathrm{N}$ & $\mathrm{N}$ & $\mathrm{N}$ & $\mathrm{N}$ \\
\hline 3 & Daporijo & & - & - & - & - & - & - & - & - & - & - & - & - & - \\
\hline 4 & Itanagar & & $\mathrm{N}$ & $\mathrm{N}$ & $\mathrm{N}$ & $\mathrm{N}$ & $\mathrm{N}$ & $\mathrm{N}$ & $\mathrm{N}$ & $Y-(-0.39)$ & $\mathrm{N}$ & $\mathrm{N}$ & $\mathrm{N}$ & $\mathrm{N}$ & $\mathrm{N}$ \\
\hline 5 & Passighat & & $Y-(-0.14)$ & $\mathrm{N}$ & $\mathrm{N}$ & $\mathrm{N}$ & $\mathrm{N}$ & $\mathrm{N}$ & $\mathrm{N}$ & $\mathrm{N}$ & $\mathrm{N}$ & $\mathrm{N}$ & $\mathrm{N}$ & $\mathrm{N}$ & $\mathrm{N}$ \\
\hline 6 & Roing & & $\mathrm{N}$ & $\mathrm{N}$ & $\mathrm{N}$ & $\mathrm{N}$ & $\mathrm{N}$ & $\mathrm{N}$ & $\mathrm{N}$ & $\mathrm{N}$ & $\mathrm{N}$ & $\mathrm{N}$ & $\mathrm{N}$ & $\mathrm{N}$ & $\mathrm{N}$ \\
\hline 7 & Seppa & & - & - & - & - & - & - & - & - & - & - & - & - & - \\
\hline 8 & Ziro & & $\mathrm{N}$ & $Y+(6.17)$ & $Y+(1.86)$ & $\mathrm{N}$ & $\mathrm{N}$ & $\mathrm{N}$ & $\mathrm{N}$ & $\mathrm{N}$ & $\mathrm{N}$ & $Y-(-1.40)$ & $\mathrm{N}$ & $\mathrm{N}$ & $\mathrm{N}$ \\
\hline 9 & Chaparmukh & \multirow{11}{*}{ AS } & - & - & - & - & - & - & - & - & - & - & - & - & 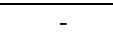 \\
\hline 10 & Dibrugarh & & $Y-(-0.12)$ & $\mathrm{N}$ & $\mathrm{N}$ & $\mathrm{N}$ & $\mathrm{N}$ & $\mathrm{N}$ & $\mathrm{N}$ & $\mathrm{N}$ & $\mathrm{N}$ & $Y-(0.15)$ & $Y-(-0.20)$ & $Y-(-0.32)$ & $Y-(-0.06)$ \\
\hline 11 & Dhubri & & $\mathrm{N}$ & $Y+(0.16)$ & $Y+(0.48)$ & $\mathrm{Y}+(0.49)$ & $\mathrm{Y}+(0.11)$ & $\mathrm{N}$ & $\mathrm{Y}+(0.07)$ & $\mathrm{N}$ & $\mathrm{N}$ & $\mathrm{N}$ & $\mathrm{N}$ & $\mathrm{N}$ & $Y+(0.13)$ \\
\hline 12 & Guwahati & & $Y+(0.07)$ & $Y+(0.14)$ & $Y+(0.15)$ & $\mathrm{N}$ & $\mathrm{N}$ & $\mathrm{N}$ & $\mathrm{N}$ & $\mathrm{N}$ & $\mathrm{N}$ & $\mathrm{N}$ & $\mathrm{N}$ & $\mathrm{N}$ & $Y+(0.03)$ \\
\hline 13 & Jorhat & & $\mathrm{N}$ & $\mathrm{N}$ & $\mathrm{N}$ & $\mathrm{N}$ & $\mathrm{N}$ & $\mathrm{N}$ & $\mathrm{N}$ & $\mathrm{N}$ & $\mathrm{N}$ & $\mathrm{N}$ & $\mathrm{N}$ & $\mathrm{N}$ & $\mathrm{N}$ \\
\hline 14 & Kumbhigram & & $\mathrm{N}$ & $\mathrm{N}$ & $Y+(0.60)$ & $\mathrm{N}$ & $\mathrm{N}$ & $\mathrm{Y}-(-0.13)$ & $\mathrm{N}$ & $\mathrm{N}$ & $\mathrm{N}$ & $\mathrm{N}$ & $\mathrm{N}$ & $\mathrm{N}$ & $\mathrm{N}$ \\
\hline 15 & Likabali & & - & - & - & - & - & - & - & - & - & - & - & - & - \\
\hline 16 & Mohanbari & & $\mathrm{N}$ & $\mathrm{N}$ & $\mathrm{N}$ & $\mathrm{N}$ & $\mathrm{N}$ & $\mathrm{N}$ & $\mathrm{N}$ & $\mathrm{N}$ & $\mathrm{N}$ & $\mathrm{N}$ & $\mathrm{N}$ & $\mathrm{N}$ & $\mathrm{N}$ \\
\hline 17 & N. Lakhimpur & & $\mathrm{N}$ & $\mathrm{N}$ & $Y+(0.17)$ & $\mathrm{N}$ & $\mathrm{N}$ & $Y+(0.14)$ & $Y+(0.07)$ & $Y+(0.13)$ & $\mathrm{N}$ & $\mathrm{N}$ & $\mathrm{N}$ & $\mathrm{N}$ & $\mathrm{N}$ \\
\hline 18 & Silchar & & $\mathrm{N}$ & $\mathrm{N}$ & $N$ & $\mathrm{~N}$ & $\mathrm{~N}$ & $Y+(0.25)$ & $N$ & $Y+(0.37)$ & $Y+(0.25)$ & $\mathrm{Y}+(0.28)$ & $Y+(0.50)$ & $Y+(0.40)$ & $Y+(0.28)$ \\
\hline 19 & Tezpur & & $\mathrm{N}$ & $\mathrm{N}$ & $\mathrm{N}$ & $\mathrm{N}$ & $\mathrm{N}$ & $\mathrm{N}$ & $\mathrm{N}$ & $\mathrm{N}$ & $\mathrm{N}$ & $\mathrm{N}$ & $\mathrm{N}$ & $\mathrm{N}$ & $\mathrm{N}$ \\
\hline 20 & Imphal & $\mathrm{MN}$ & $\mathrm{N}$ & $\mathrm{N}$ & $Y+(0.23)$ & $\mathrm{N}$ & $\mathrm{Y}+(0.14)$ & $\mathrm{N}$ & $\mathrm{Y}+(0.09)$ & $Y+(0.07)$ & $\mathrm{Y}+(0.07)$ & $\mathrm{N}$ & $\mathrm{N}$ & $\mathrm{Y}+(0.17)$ & $Y+(0.08)$ \\
\hline 21 & Barapani & \multirow{4}{*}{$\mathrm{ML}$} & - & - & - & - & - & - & - & - & - & - & - & - & - \\
\hline 22 & Cherrapunji & & $\mathrm{N}$ & $\mathrm{N}$ & $\mathrm{N}$ & $\mathrm{N}$ & $\mathrm{N}$ & $\mathrm{N}$ & $\mathrm{N}$ & $\mathrm{N}$ & $\mathrm{N}$ & $\mathrm{N}$ & $\mathrm{N}$ & $\mathrm{N}$ & $\mathrm{N}$ \\
\hline 23 & Shillong & & $Y+(0.28)$ & $Y+(0.34)$ & $Y+(0.37)$ & $Y+(0.20)$ & $\mathrm{N}$ & $\mathrm{N}$ & $\mathrm{N}$ & $Y+(0.07)$ & $\mathrm{Y}+(0.12)$ & $Y+(0.30)$ & $\mathrm{N}$ & $\mathrm{Y}+(0.53)$ & $Y+(0.20)$ \\
\hline 24 & Tura & & $Y-(-8.63)$ & $\mathrm{N}$ & $N$ & $Y-(-7.69)$ & $\mathrm{N}$ & $Y-(-5.00)$ & Y- $(-3.88)$ & $Y-(-2.96)$ & $N$ & $\mathrm{~N}$ & $\mathrm{~N}$ & $\mathrm{~N}$ & $\mathrm{~N}$ \\
\hline 25 & Aizawl & \multirow{2}{*}{$\mathrm{MZ}$} & - & - & - & - & - & - & - & - & - & - & - & - & - \\
\hline 26 & Lengpui & & $Y-(-1.57)$ & $\mathrm{N}$ & $\mathrm{N}$ & $\mathrm{N}$ & $\mathrm{N}$ & $\mathrm{N}$ & $\mathrm{N}$ & $\mathrm{N}$ & $\mathrm{N}$ & $\mathrm{N}$ & $\mathrm{N}$ & $\mathrm{N}$ & $\mathrm{N}$ \\
\hline 27 & Dimapur & \multirow{2}{*}{ NL } & $\mathrm{N}$ & $\mathrm{N}$ & $\mathrm{N}$ & $\mathrm{N}$ & $\mathrm{N}$ & $\mathrm{N}$ & $\mathrm{N}$ & $\mathrm{N}$ & $\mathrm{N}$ & $\mathrm{N}$ & $\mathrm{N}$ & $Y+(0.43)$ & $\mathrm{N}$ \\
\hline 28 & Kohima & & $\mathrm{N}$ & $Y+(0.71)$ & $\mathrm{N}$ & $\mathrm{N}$ & $\mathrm{N}$ & $\mathrm{N}$ & $\mathrm{N}$ & $\mathrm{N}$ & $\mathrm{N}$ & $\mathrm{N}$ & $\mathrm{N}$ & $\mathrm{N}$ & $\mathrm{N}$ \\
\hline 29 & Agartala & \multirow{2}{*}{ TR } & $Y+(0.42)$ & $Y+(0.30)$ & $Y+(0.29)$ & $\mathrm{N}$ & $\mathrm{N}$ & $\mathrm{N}$ & $\mathrm{N}$ & $\mathrm{N}$ & $\mathrm{N}$ & $\mathrm{Y}+(0.06)$ & $Y+(0.13)$ & $Y+(0.24)$ & $Y+(0.12)$ \\
\hline 30 & Kailashahar & & $Y+(0.08)$ & $\mathrm{N}$ & $\mathrm{N}$ & $\mathrm{N}$ & $\mathrm{N}$ & $\mathrm{N}$ & $\mathrm{N}$ & $Y-(-0.06)$ & $\mathrm{N}$ & $\mathrm{N}$ & $\mathrm{N}$ & $\mathrm{N}$ & $\mathrm{N}$ \\
\hline \multirow{2}{*}{\multicolumn{2}{|c|}{$\begin{array}{l}\text { No. of stations with } \mathrm{Y}+ \\
\text { No. of stations with } \mathrm{Y} \text { - }\end{array}$}} & & 4 & 6 & 8 & 2 & 2 & 2 & 3 & 4 & 3 & 3 & 2 & 5 & 6 \\
\hline & & & 4 & 0 & 0 & 1 & 0 & 2 & 1 & 3 & 0 & 2 & 1 & 1 & 1 \\
\hline
\end{tabular}


Table 13. State and region-wise MK test results and Sen slopes $\left(\%\right.$ year $\left.^{-1}\right)$ for monthly and yearly average relative humidity

\begin{tabular}{|c|c|c|c|c|c|c|c|c|c|c|c|c|c|}
\hline State & Jan & Feb & Mar & Apr & May & Jun & Jul & Aug & Sep & Oct & Nov & Dec & Yearly \\
\hline Arunachal Pradesh & $\mathrm{N}$ & $\mathrm{N}$ & $\mathrm{N}$ & $\mathrm{N}$ & $\mathrm{N}$ & $\mathrm{N}$ & $\mathrm{N}$ & $\mathrm{N}$ & $\mathrm{N}$ & $\mathrm{N}$ & $\mathrm{N}$ & $\mathrm{N}$ & $\mathrm{N}$ \\
\hline Assam & $\mathrm{N}$ & $\mathrm{N}$ & $Y+(0.17)$ & $\mathrm{N}$ & $\mathrm{N}$ & $\mathrm{N}$ & $\mathrm{N}$ & $\mathrm{N}$ & $\mathrm{N}$ & $\mathrm{N}$ & $\mathrm{N}$ & $\mathrm{N}$ & $\mathrm{N}$ \\
\hline Manipur & $\mathrm{N}$ & $\mathrm{N}$ & $Y+(0.23)$ & $\mathrm{N}$ & $Y+(0.14)$ & $\mathrm{N}$ & $\mathrm{Y}+(0.09)$ & $\mathrm{Y}+(0.07)$ & $Y+(0.07)$ & $\mathrm{N}$ & $\mathrm{N}$ & $Y+(0.17)$ & $Y+(0.08)$ \\
\hline Meghalaya & $\mathrm{N}$ & $\mathrm{N}$ & $Y+(0.20)$ & $\mathrm{N}$ & $\mathrm{N}$ & $\mathrm{N}$ & $\mathrm{N}$ & $\mathrm{N}$ & $\mathrm{N}$ & $\mathrm{N}$ & $\mathrm{N}$ & $\mathrm{N}$ & $\mathrm{N}$ \\
\hline Mizoram & $Y-(-1.57)$ & $\mathrm{N}$ & $\mathrm{N}$ & $\mathrm{N}$ & $\mathrm{N}$ & $\mathrm{N}$ & $\mathrm{N}$ & $\mathrm{N}$ & $\mathrm{N}$ & $\mathrm{N}$ & $\mathrm{N}$ & $\mathrm{N}$ & $\mathrm{N}$ \\
\hline Nagaland & $\mathrm{N}$ & $\mathrm{N}$ & $\mathrm{N}$ & $Y-(-0.78)$ & $Y-(-0.57)$ & $Y-(-0.36)$ & $Y-(-0.33)$ & $\mathrm{N}$ & $\mathrm{N}$ & $\mathrm{N}$ & $Y+(0.34)$ & $\mathrm{N}$ & $\mathrm{N}$ \\
\hline Tripura & $Y+(0.25)$ & $\mathrm{Y}+(0.19)$ & $\mathrm{Y}+(0.16)$ & $\mathrm{N}$ & $\mathrm{N}$ & $\mathrm{N}$ & $\mathrm{N}$ & $\mathrm{N}$ & $\mathrm{N}$ & $\mathrm{N}$ & $\mathrm{N}$ & $Y+(0.13)$ & $Y+(0.06)$ \\
\hline NE India & $\mathrm{N}$ & $\mathrm{N}$ & $\mathrm{Y}+(0.13)$ & $\mathrm{N}$ & $\mathrm{Y}-(-0.11)$ & $\mathrm{N}$ & $\mathrm{N}$ & $\mathrm{N}$ & $\mathrm{N}$ & $\mathrm{N}$ & $\mathrm{N}$ & $\mathrm{N}$ & $\mathrm{N}$ \\
\hline
\end{tabular}

Table 14. Station-wise MK test results and Sen slopes $\left(\mathrm{km} \mathrm{h}^{-1}\right.$ year $\left.{ }^{-1}\right)$ for monthly and yearly average wind speed

\begin{tabular}{|c|c|c|c|c|c|c|c|c|c|c|c|c|c|c|c|}
\hline Sl. No. & Station & State & Jan & Feb & Mar & Apr & May & Jun & Jul & Aug & Sep & Oct & Nov & Dec & Yearly \\
\hline 1 & Aalo & \multirow{8}{*}{ AP } & - & - & - & - & - & - & - & - & - & - & - & - & - \\
\hline 2 & Basar & & - & - & - & - & - & - & - & - & - & - & - & - & - \\
\hline 3 & Daporijo & & - & - & - & - & - & - & - & - & - & - & - & - & - \\
\hline 4 & Itanagar & & - & - & - & - & - & - & - & - & - & - & - & - & - \\
\hline 5 & Passighat & & - & - & - & - & - & - & - & - & - & - & - & - & - \\
\hline 6 & Roing & & - & - & - & - & - & - & - & - & - & - & - & - & - \\
\hline 7 & Seppa & & - & - & - & - & - & - & - & - & - & - & - & - & - \\
\hline 8 & Ziro & & $\mathrm{N}$ & $\mathrm{N}$ & $\mathrm{N}$ & $\mathrm{N}$ & $\mathrm{N}$ & $\mathrm{N}$ & $\mathrm{N}$ & $\mathrm{N}$ & $\mathrm{N}$ & $\mathrm{N}$ & $\mathrm{N}$ & $\mathrm{N}$ & $\mathrm{N}$ \\
\hline 9 & Chaparmukh & \multirow{11}{*}{ AS } & Y- $(-0.08)$ & Y- $(-0.11)$ & $\mathrm{Y}-(-0.12)$ & $\mathrm{N}$ & $\mathrm{N}$ & $\mathrm{N}$ & Y- $(-0.06)$ & $Y-(-0.05)$ & $\mathrm{N}$ & $\mathrm{Y}-(-0.11)$ & $\mathrm{N}$ & $Y-(-0.04)$ & $Y-(-0.05)$ \\
\hline 10 & Dibrugarh & & $Y-(-0.05)$ & $Y-(-0.08)$ & $Y-(-0.07)$ & $Y-(-0.08)$ & $\mathrm{N}$ & $Y-(-0.06)$ & $Y-(-0.09)$ & $Y-(-0.06)$ & $\mathrm{N}$ & $\mathrm{N}$ & $Y-(-0.05)$ & $Y-(-0.04)$ & $Y-(-0.05)$ \\
\hline 11 & Dhubri & & $\mathrm{N}$ & $N$ & $N$ & $N$ & $\mathrm{~N}$ & $N$ & $\mathrm{~N}$ & $\mathrm{~N}$ & $\mathrm{~N}$ & $N$ & $\mathrm{~N}$ & $N$ & $N$ \\
\hline 12 & Guwahati & & - & - & - & - & - & - & - & - & - & - & - & - & - \\
\hline 13 & Jorhat & & - & - & - & - & - & - & - & - & - & - & - & - & - \\
\hline 14 & Kumbhigram & & - & - & - & - & - & - & - & - & - & - & - & - & - \\
\hline 15 & Likabali & & - & - & - & - & - & - & - & - & - & - & - & - & - \\
\hline 16 & Mohanbari & & - & - & - & - & - & - & - & - & - & - & - & - & - \\
\hline 17 & N. Lakhimpur & & - & - & - & - & - & - & - & - & - & - & - & - & - \\
\hline 18 & Silchar & & - & - & - & - & - & - & - & - & - & - & - & - & - \\
\hline 19 & Tezpur & & $\mathrm{N}$ & $\mathrm{N}$ & $\mathrm{N}$ & $\mathrm{N}$ & $\mathrm{N}$ & $\mathrm{N}$ & $\mathrm{N}$ & $\mathrm{N}$ & $\mathrm{N}$ & $\mathrm{N}$ & $\mathrm{N}$ & $\mathrm{N}$ & $\mathrm{N}$ \\
\hline 20 & Imphal & $\mathrm{MN}$ & $\mathrm{N}$ & $\mathrm{N}$ & $\mathrm{N}$ & $\mathrm{N}$ & $Y-(-0.05)$ & $\mathrm{N}$ & $\mathrm{N}$ & $\mathrm{N}$ & $\mathrm{N}$ & $\mathrm{N}$ & $\mathrm{N}$ & $\mathrm{N}$ & $\mathrm{N}$ \\
\hline 21 & Barapani & \multirow{4}{*}{$\mathrm{ML}$} & - & - & - & - & - & - & - & - & - & - & - & - & - \\
\hline 22 & Cherrapunji & & $\mathrm{N}$ & $\mathrm{N}$ & $\mathrm{N}$ & $\mathrm{N}$ & $\mathrm{N}$ & $\mathrm{N}$ & $\mathrm{N}$ & $\mathrm{N}$ & $\mathrm{N}$ & $\mathrm{N}$ & $\mathrm{N}$ & $\mathrm{N}$ & $\mathrm{N}$ \\
\hline 23 & Shillong & & $\mathrm{N}$ & $\mathrm{N}$ & $Y+(0.07)$ & $\mathrm{N}$ & $\mathrm{N}$ & $\mathrm{N}$ & $\mathrm{N}$ & $\mathrm{N}$ & $\mathrm{N}$ & $\mathrm{N}$ & $\mathrm{N}$ & $\mathrm{N}$ & $\mathrm{N}$ \\
\hline 24 & Tura & & $\mathrm{Y}-(-0.39)$ & $Y-(-0.53)$ & $\mathrm{N}$ & $\mathrm{N}$ & $Y-(-0.50)$ & $Y-(-0.50)$ & $Y-(-0.46)$ & $Y-(-0.35)$ & $Y-(-0.42)$ & $Y-(-0.35)$ & $\mathrm{N}$ & $\mathrm{N}$ & $\mathrm{N}$ \\
\hline 25 & Aizawl & \multirow{2}{*}{$\mathrm{MZ}$} & $\mathrm{N}$ & $\mathrm{N}$ & $\mathrm{N}$ & $\mathrm{N}$ & $\mathrm{N}$ & $\mathrm{N}$ & $\mathrm{N}$ & $\mathrm{N}$ & $\mathrm{N}$ & $\mathrm{N}$ & $\mathrm{N}$ & $\mathrm{N}$ & $\mathrm{N}$ \\
\hline 26 & Lengpui & & - & - & - & - & - & - & - & - & - & - & - & - & - \\
\hline 27 & Dimapur & \multirow{2}{*}{ NL } & - & - & - & - & - & - & - & - & - & - & - & - & - \\
\hline 28 & Kohima & & $Y+(1.00)$ & $\mathrm{Y}+(1.20)$ & $Y+(1.44)$ & $\mathrm{N}$ & $\mathrm{N}$ & $\mathrm{N}$ & $\mathrm{N}$ & $\mathrm{N}$ & $\mathrm{N}$ & $\mathrm{N}$ & $\mathrm{N}$ & $\mathrm{N}$ & $\mathrm{N}$ \\
\hline 29 & Agartala & \multirow[b]{2}{*}{ TR } & $\mathrm{Y}-(-0.06)$ & $Y-(-0.10)$ & $\mathrm{Y}-(-0.13)$ & $Y-(-0.19)$ & $\mathrm{Y}-(-0.15)$ & $\mathrm{Y}-(-0.11)$ & $Y-(-0.13)$ & $Y-(-0.13)$ & $Y-(-0.08)$ & $Y-(-0.07)$ & $\mathrm{Y}-(-0.07)$ & $Y-(-0.04)$ & $\mathrm{Y}-(-0.11)$ \\
\hline 30 & Kailashahar & & $\mathrm{N}$ & $\mathrm{N}$ & $N$ & Y- $(-0.07)$ & N & $\mathrm{N}$ & $\mathrm{N}$ & $\mathrm{N}$ & $\mathrm{N}$ & $\mathrm{N}$ & $\mathrm{N}$ & N & $\mathrm{N}$ \\
\hline \multirow{2}{*}{\multicolumn{2}{|c|}{$\begin{array}{l}\text { No. of stations with } \mathrm{Y}+ \\
\text { No. of stations with } \mathrm{Y} \text { - }\end{array}$}} & & 1 & 1 & 2 & 0 & 0 & 0 & 0 & 0 & 0 & 0 & 0 & 0 & 0 \\
\hline & & & 4 & 4 & 3 & 3 & 3 & 3 & 4 & 4 & 2 & 3 & 2 & 3 & 3 \\
\hline
\end{tabular}


Table 15. State and region-wise MK test results and Sen slopes $\left(\mathrm{km} \mathrm{h}^{-1}\right.$ year $\left.{ }^{-1}\right)$ for monthly and yearly average wind speed

\begin{tabular}{|c|c|c|c|c|c|c|c|c|c|c|c|c|c|}
\hline State & Jan & Feb & Mar & Apr & May & Jun & Jul & Aug & Sep & Oct & Nov & Dec & Yearly \\
\hline Arunachal Pradesh & $\mathrm{N}$ & $\mathrm{N}$ & $\mathrm{N}$ & $\mathrm{N}$ & $\mathrm{N}$ & $\mathrm{N}$ & $\mathrm{N}$ & $\mathrm{N}$ & $\mathrm{N}$ & $\mathrm{N}$ & $\mathrm{N}$ & $\mathrm{N}$ & $\mathrm{N}$ \\
\hline Assam & $Y-(-0.07)$ & $Y-(-0.05)$ & $\mathrm{N}$ & $Y-(-0.09)$ & N & $Y-(-0.07)$ & $Y-(-0.08)$ & $Y-(-0.08)$ & $Y-(-0.06)$ & $Y-(-0.08)$ & $Y-(-0.10)$ & $Y-(-0.07)$ & $Y-(-0.07)$ \\
\hline Manipur & $\mathrm{N}$ & $\mathrm{N}$ & $\mathrm{N}$ & $\mathrm{N}$ & $Y-(-0.05)$ & $\mathrm{N}$ & $\mathrm{N}$ & $\mathrm{N}$ & $\mathrm{N}$ & $\mathrm{N}$ & $\mathrm{N}$ & $\mathrm{N}$ & $\mathrm{N}$ \\
\hline Meghalaya & $\mathrm{N}$ & $\mathrm{N}$ & $Y+(0.07)$ & $\mathrm{N}$ & $\mathrm{N}$ & $\mathrm{N}$ & $\mathrm{N}$ & $\mathrm{N}$ & $\mathrm{N}$ & $\mathrm{N}$ & $\mathrm{N}$ & $\mathrm{N}$ & $\mathrm{N}$ \\
\hline Mizoram & $\mathrm{N}$ & $\mathrm{N}$ & $\mathrm{N}$ & $\mathrm{N}$ & $\mathrm{N}$ & $\mathrm{N}$ & $\mathrm{N}$ & $\mathrm{N}$ & $\mathrm{N}$ & $\mathrm{N}$ & $\mathrm{N}$ & $\mathrm{N}$ & $\mathrm{N}$ \\
\hline Nagaland & $Y+(1.00)$ & $Y+(1.20)$ & $Y+(1.44)$ & $\mathrm{N}$ & $\mathrm{N}$ & $\mathrm{N}$ & $\mathrm{N}$ & $\mathrm{N}$ & $\mathrm{N}$ & $\mathrm{N}$ & $\mathrm{N}$ & $\mathrm{N}$ & $\mathrm{N}$ \\
\hline Tripura & $Y-(-0.03)$ & Y- $(-0.05)$ & $Y-(-0.06)$ & $Y-(-0.14)$ & $Y-(-0.04)$ & $\mathrm{N}$ & $Y-(-0.07)$ & $Y-(-0.06)$ & $Y-(-0.05)$ & $\mathrm{N}$ & $Y-(-0.03)$ & $Y-(-0.03)$ & $Y-(-0.05)$ \\
\hline NE India & $\mathrm{Y}-(-0.05)$ & $\mathrm{N}$ & $\mathrm{N}$ & $\mathrm{N}$ & $\mathrm{N}$ & $\mathrm{N}$ & $Y-(-0.10)$ & $Y-(-0.09)$ & $\mathrm{Y}-(-0.07)$ & $\mathrm{Y}-(-0.06)$ & $\mathrm{Y}-(-0.06)$ & $Y-(-0.05)$ & $Y-(-0.05)$ \\
\hline
\end{tabular}


Data for mean air temperature could not be derived at three stations (Aizawl, Daporijo and Roing) and hence these were excluded from this analysis. Similar to minimum temperature, in this case also, the number of stations with significant positive trend was higher than that with rarely detected significant negative trend. The only station Imphal in Manipur had significant increasing trend in all the months. All the stations tested to have no significant trend in April except Imphal. The yearly average mean temperature was also found to be significantly increasing in 11 stations.

When analyzed state-wise (Table 11), Manipur showed significant increasing trend in all the months followed by Tripura with nine months. In the annual assessment also, Manipur and Tripura were detected with significant increasing trend with highest slope of $0.04{ }^{\circ} \mathrm{C}$ per year at Manipur. No significant falling trend was not found in any of the states. When considered the NE India as a whole, five months as well as the yearly assessment showed significant rising trend with highest slope of $0.04{ }^{\circ} \mathrm{C}$ per year in September.

\subsection{Mean relative humidity}

Table 12 displays the MK test results on mean relative humidity for 24 stations, along with the Sen slope values corresponding to significant trends. Six stations (Daporijo, Seppa, Chaparmukh, Likabali, Barapani, and Aizwal) had no observed relative humidity data and had to be excluded from this analysis. In all months, the number of stations with no significant trend was maximum, and then, in most months, the number of stations with significant increasing trend was more than that with significant negative trend.

In state level (Table 13), Manipur recorded significant increasing trend in six months followed by Tripura with four months. Both Manipur and Tripura were detected with significant increasing trend in the yearly assessment also. Nagaland reported significant decreasing trend in mean relative humidity during April-July. Considering NE India as a whole, March was found to have significant increasing trend, whereas, May was detected with significant decreasing trend.

\subsection{Wind speed}

Table 14 displays the MK test results of wind speed, along with the Sen slope values for significant trends. Only 13 stations had wind speed data and were considered in this analysis. Overall, maximum number of stations were detected to have no significant trend. Other than that, more number of stations experienced significant decreasing trend in wind speed compared to increasing trend, which was found in 1-2 stations during JanuaryMarch only.

Considering state-averages (Table 15), Assam and Tripura were detected to have significant decreasing trend in 10 months each. Understandably, these two states showed significant decreasing trend in the yearly assessment also. When considered the entire NE India as a whole, significant decreasing trend was detected during July-January, as well as in the yearly assessment.

\section{Conclusions}

Availability of accurate meteorological data in NE India is very limited. In this study, the authors have tried to collect meteorological data from reliable government agencies for as many stations as available within the study area.

Among the $\mathbf{3 0}$ meteorological stations considered in this study, both statistically significant increasing as well as decreasing trends were found in rainfall for most months during 1971-2010. Most of the negative trends were found during July-August (monsoon), whereas, maximum stations with positive trends were detected in April-May (pre-monsoon), and October-November (post-monsoon). However, in annual rainfall, no station reported any significant trend except Dhubri. This may indicate interseasonal shifting of rainfall without much change in the annual total rainfall. In case of number of rainy days, most of the positive trends were found during March-May (premonsoon) and negative trends were found during September-December. In case of temperatures, all maximum, minimum and mean temperatures showed similar results with more number of stations having significant rising trends compared to that having significant falling trends. However, the bias was more prominent in cases of minimum and mean temperatures. In some stations, minimum temperature was also found to be increasing throughout the year. Some major towns like Guwahati, Imphal, Agartala and Kailashshahar showed significant positive trends in mean temperature mostly during June-December. Highest positive Sen slope value was found to be $0.28{ }^{\circ} \mathrm{C}$ per year at Seppa October, whereas, highest negative Sen slope was $-0.34{ }^{\circ} \mathrm{C}$ per year at Tura in March. Mean relative humidity was, in general, found to be significantly increasing, especially during February-March. In some stations, wind speed was found to have significant negative trends in almost all the months, with Agartala being the most affected.

The indication of seasonal shifting of rainfall may adversely affect the agricultural activities of NE India, which is mainly rainfed in nature. In addition, many stations show significant increasing trend in temperature, especially minimum temperature, which will surely affect the water balance of the region in near future, demanding a review of the cropping pattern and crop species.

\section{Acknowledgement}

This study is sponsored by Indian National Committee on Surface Water, Ministry of Water Resources, Govt. of India (Grant No. 23/73/2012-R\&D). The authors would also like to express their appreciation to India Meteorological Department and State Govt. Departments of seven North East States of India for providing the meteorological data.

\section{References}

Bandyopadhyay A., Bhadra A., Raghuwanshi N.S. and Singh R. (2009), Temporal trends in estimates of reference evapotranspiration over India, Journal of Hydrologic Engineering, 14(5), 508-515.

Bandyopadhyay A., Pal A. and Debnath S. (2011), Development of an ArcGIS Toolbar for Trend Analysis of Climatic Data. Proceedings of International Conference on Agricultural, 
Biotechnology, Biosystems \& Biological Engineering (ABBBE) 2011, Phuket, Thailand, December 21-23, 2011, WASET, 60: 563-570.

Das P.J. (2004), Rainfall regime of northeast India: A hydrometeorological study with special emphasis on the Brahmaputra basin. Unpublished Ph.D. Thesis, Gauhati University, Guwahati.

Das A., Ghosh P.K., Choudhury B.U., Patel D.P., Munda G.C., Ngachan S.V. and Chowdhury P. (2009), Climate change in north east India: recent facts and events - worry for agricultural management. Proceedings of Workshop on Impact of Climate Change on Agriculture, ISPRS Archives XXXVIII-8/W3 Workshop, Ahmedabad, December 17-19, 2009: 32-37.

De U.S. and Mukhopadhyay R.K. (1998), Severe heat wave over the Indian subcontinent in 1998, in perspective of global climate, Current Science, 75(12), 1308-1311.

Dietz E.J. and Killeen T.J. (1981), A non parametric multivariate test for monotone trend with pharmaceutical applications, Journal of American Statistical Association, 76, 169-174.

Goswami D.C. and Das P.J. (2003), The Brahmaputra river, India: The eco-hydrological context of water use in one of world's most unique river systems, Ecologist Asia, Special issue on large dams in northeast India - Rivers, forests, people and power, 11(1), 9-14.

Hamed K.H. and Rao A.R. (1998), A modified Mann-Kendall trend test for autocorrelated data, Journal of Hydrology, 204, 182-196.

Hingane L.S., Rupakumar K. and Ramanamurthy B.V. (1985), Long term needs of surface air temperature in India, Journal of Climatology, 5, 521-528.

Hirsch R.M., Slack J.R. and Smith R.A. (1982), Techniques of trend analysis for monthly water quality data, Water Resources Research, 18(1), 107-121.

IPCC. 2001. Climate change 2001: The scientific basis. Contribution of working group I to the third assessment report of the Intergovernmental Panel on Climate Change. Cambridge University Press, Cambridge, UK.

IPCC. 2007. Climate Change 2007: Impacts, adaptation and vulnerability. Contribution of working group II to the fourth assessment report of the Intergovernmental Panel on Climate Change, M.L. Parry, O.F. Canziani, J.P. Palutikof, P.J. Van der Linden and C.E. Hanson, (Eds.) Cambridge, UK: Cambridge University Press.

Kendall, M.G. 1975. Rank correlation methods. Charles Griffin, Page 202.

Lettenmaier D.P. (1988), Multivariate non parametric tests for trend in water quality, Water Resources Bulletin, 24(3), 505-512.

Libiseller C. and Grimvall A. (2002), Performance of partial MannKendall tests for trend detection in the presence of covariates, Environmetrics, 13, 71-84.

Mann H.B. (1945), Non-parametric test against trend, Econometrika, 13, 245-259.

Matalas N.C. and Langbein W.B. (1962), Information content of the mean, Journal of Geophysical Research, 67(9), 3441-3448.

Mirza M.M.Q., Warrick R.A., Ericksen N.J. and Kenny G.J. (1998), Trends and persistence in precipitation in the Ganges, Brahmaputra and Meghna river basins, Hydrological SciencesJournal-des Hydrologiques, 43(6), 845-858.
Nema M.K., Jain S.K. and Mishra P.K. (2014), Spatial and temporal distribution of rainfall trend in Bist-Doab region of Punjab. Proc., 19th International Conference on "Hydraulics, Water Resources, Coastal and Environmental Engineering", Bhopal, India, December 18-20, 2014: 285-290.

Pant G.B., Rupakumar K. and Borgaonkar H.P. (1999), Climate and its long-term variability over the western Himalaya during the past two centuries. In: Dash S.K. and Bahadur, J. (Eds.). The Himalayan Environment, New Age International (P) Limited, Publishers, New Delhi: 172-184.

Pant G.B. and Rupakumar K. 1997. Climates of south Asia. John Wiley and Sons, New York.

Paramanik S.K. and Jagannathan P. (1954), Climate change in India (II)-Temperature, Indian Journal of Meteorology and Geophysics, 5, 1-19.

Rupakumar K., Krishnakumar K. and Pant G.B. (1994), Diurnal asymmetry of surface air temperature trends over India, Geophysics Research Letters, 15, 677-680.

Salas J.D., Delleur J.W., Yevjevich V. and Lane W.L. (1980), Applied modelling of hydrologic time series. Water Resource Publications, Littleton Co., USA.

Sen P.K. (1968), Estimates of the regression coefficient based on Kendall's tau, Journal of American Statistical Association, 63, 1379-1389.

Singh N. and Sontakke N.A. (2002), On climatic fluctuations and environmental changes of the Indo-Gangetic plains, India, Climatic Change, 52, 287-313.

Singh R.S., Narain P. and Sharma K.D. (2001), Climate changes in Luni river basin of arid western Rajasthan (India), Vayu Mandal, 31(1-4), 103-106.

Srivastava H.N., Dewan B.N., Dikshit S.K., Rao G.S.P., Singh S.S. and Rao R. (1992), Decadal trends in Climate over India, Mausam, 43, 7-20.

Udo-Inyang U.C. and Edem I.D. (2012), Analysis of rainfall trends in Akwalbom state, Nigeria, Journal of Environment and Earth Science, 2(8), 60-71.

Yue S. and Wang C.Y. (2004), The Mann-Kendall test modified by effective sample size to detect trend in serially correlated hydrological series, Water Resources Management, 18, 201-218. 